\title{
ON THE BOUNDARY THEORY FOR MARKOV CHAINS
}

BY

KAI LAI CHUNG

Stanford University, U.S.A. (1)

\section{§ 1. Introduction and Summary}

The boundary theory of Markov chains, as viewed here, is the study of essential discontinuities (viz., those which are not jumps) of the sample functions. The underlying assumptions are such that these discontinuities form a set of measure zero on the time axis and that for any given time $t$, the sample function will almost certainly have only jumps within an open interval containing $t$, reaching the boundary at both ends if at all. Thus it is a question of "how the sample curves manage to go to infinity and to come back from there" (see the preface to [1]). In Paul Lévy's terminology [9], it is a study of "fictitious states". Depending on whether the transition is to or from such a state, it is called a point on the "exit" or "entrance" boundary by Feller ([6], [7]). These ideal boundaries can be formally defined in terms of the R. S. Martin boundary theory (see [4], [5], and [8]), and the question becomes that of a suitable compactification of a discrete set, the denumerable state space of the Markov chain.

In this paper we are mainly concerned with the probabilistico-analytical aspect of the theory rather than the algebraico-topological one, if such a rough distinction may be made. Although the boundary can be defined in the general case and in more than one way, so far only the atomic part consisting of a denumerable number of boundary points has been penetrated in any sense, and substantially so only if their number is finite. It is this part which engages our attention here.

The content of this paper is most directly related to Feller's pioneering work [7]. Indeed, part of the present work arose from an effort to clarify and consolidate his results in probabilistic terms. While Feller regards his problem as one of constructing

(1) This research is supported in part by the Office of Scientific Research of the United States Air Force. 
Markovian transition matrices out of simpler elements, here the viewpoint is that of analyzing such given matrices and their associated processes. It is perhaps a logical truism to say that a complete construction is tantamount to a complete analysis, but there is a difference in emphasis. We take the liberty to include, particularly in $\S 6$, a number of results whose Laplace transformed versions are already in Feller's paper.

Though Feller used the language of operator theory, he has in essence created his own methods based on the resolvent equation. Reuter, in a series of papers ([12], [13] and [14]), presented the semi-group treatment of the subject and contributed to it in several respects. Neveu [11] gave a synthesis in a more general context comprising the theory of taboo states as well as boundaries. The present work has profited from the works of both authors as well as some private discussions with them and with R. S. Phillips and David Williams.

We have found it possible to derive the basic results from the first principles of probability theory together with the kind of direct methods used in [1] and [3]. Laplace transforms are employed only at a later stage. It should be mentioned that while certain analytical formulas have their "obvious" interpretations, their actual identification with probabilistic statements are not always a simple matter (see e.g. Reuter [14]). In our approach the basic quantities and their relations are obtained from considerations of the stochastic processes involved. A brief summary of the various sections will now be given.

In $\S 2$ we give as much background material as seems feasible, though some further knowledge of the subject such as contained in $\S \S$ II. 19-20 of [1] would be necessary for a thorough understanding of the paper.

In $\S 3$ the Martin boundary theory is reviewed. Since we can use only its atomic part its role is a rather formal one.

In $\S 4$ the basic theorems are derived from considerations of certain martingales, and Blackwell's theorem is invoked rather than the earlier and equivalent lattice approach of Feller [6]. The crucial link is the simple but new Theorem 4.3, which as it were connects the two sides of the boundary. The rest is an application of the strong Markov property in the form given in $\$ \S$ II. 8-9 of [1]. Theorem 4.6 and the open questions mentioned in its connection should serve as a test stone for any general theory of compactification of the state space of a Markor chain.

In $\S 5$, uncomplicated probability arguments are in evidence and the fairly general Theorem 5.5 is arrived at speedily. It gives a complete description of the sample functions when there is no accumulation of boundary points in finite time and the situation may be described as being of the renewal type. Analytically, this result 
already contains the first and easier case of Feller's construction. The idea of this approach was explained in [3] in the one-exit case and it is also one of the tools in Neveu [11] who found it independently.

In $\S 6$ we use the counterpart of Feller's idea of "canonical mapping" which amounts to an integration over time in order to convert probabilities into potentials (for nonrecurrent states). Interesting, even fruitful interpretations of the results may be obtained in this light but will not be dwelt upon here. The main result is Theorem 6.3 which yields the basic relation between the transition mechanism of a given Markov chain and its "jumping" components. This must correspond to what Feller calls a lateral condition. Theorem 6.8 gives criteria for the validity of the second (forward) system of Kolmogorov differential equations which, in contrast to the first (backward) system, is not assumed throughout.

In $\S 7$ we treat the dual chain to obtain the representation given in Theorem 7.4. This result, treated as a major consequence of our development here, is the point of departure in Feller's more algebraic theory. It must be pointed out that the dual chain is not the reversed chain (as studied in [2] in another connection) and whatever symmetry it yields is more analytic than probabilistic. However, this symmetry can be further exploited as in Neveu [11], and our lack of insistence on it may have caused some losses.

In $\S 8$ we employ the full force of Laplace transforms as completely monotonic functions. The results may be considered as furnishing some analytical insight or hindsight on the situation. In particular, Theorem 8.3 gives a criterion for complete construction under the same conditions as in Feller [7]. From this the more explicit formulas of Feller are derived with some amendment, but a full analysis of the second. case (Theorem 8.5) remains to be done.

In $\$ 9$ the one-exit case is treated in full and the results agree with those previously obtained by Reuter [13]. The connection with certain processes with independent stationary increments, discovered by Lévy [9] and analyzed by Neveu [10], is briefly mentioned.

In $\S 10$ we give an extension of the theorem of Austin-Ornstein on the positivity of the elements of a transition matrix. While the result has only peripheral contact with the present work, it is included here for its own interest.

\section{§ 2. Terminology and Notation}

We begin with a list of symbols and conventions frequently used in this paper without further explanation. They are appreciably the same as in [1] or [3], two 3-632932 Acta mathematica. 110. Imprimé le 14 octobre 1963. 
major exceptions being the omission of $\omega$ wherever possible, and the use of $f\left({ }^{1}\right)$ for $\bar{p}$. Any contrary usage will be explicitly mentioned or clearly indicated by the context.

$\mathbf{N}$ is the set of nonnegative integers. The latters $m, n$ and $\nu$ denote elements of $\mathbf{N}$.

$\mathbf{T}=[0, \infty) ; \mathbf{T}^{0}=(0, \infty)$. The letters $s, t, u$ and $v$ denote elements of $\mathbf{T}^{0}$.

$\mathbf{R}$ is the set of rational numbers in $\mathbf{T}$.

I is a denumerable set of indices. The letters $i, j$ and $k$ denote elements of $\mathbf{I}$. The letters $\theta, \theta^{\prime}, \theta^{\prime \prime}$ and $\tilde{\theta}$ denote distinct objects not in $\mathbf{I}$.

In this section a statement or formula involving an unspecified element of $\mathbf{T}^{0}$ or $\mathbf{I}$ is meant to hold for every such element. A sequence like $\left\{f_{i}\right\}$ is indexed by $\mathbf{I}$; a matrix like $\left(p_{i j}\right)$ is indexed by $\mathbf{I} \times \mathbf{I}$; a sum like $\sum$, is extended over $\mathbf{I}$. After this section, $I$ is to be replaced by $I_{\theta}$ (see below) in these conventions until further notice in $\S 6$. Actually only on rare occasions does the inclusion or exclusion of $\theta$ require a careful check.

A function is real and finite valued. A function defined on $\mathbf{T}^{0}$ and having a right-hand limit at zero is thereby extended, together with its continuity if there is, to $\mathbf{T}$.

$$
\begin{gathered}
\delta_{i j}=\left\{\begin{array}{ll}
0 & \text { if } \quad i \neq j, \\
1 & \text { if } \quad i=j .
\end{array} \quad \varepsilon(t)= \begin{cases}0 & \text { if } \quad t<0, \\
1 & \text { if } \quad t \geqslant 0 .\end{cases} \right. \\
e_{q}(t)=\left\{\begin{array}{cc}
0 & \text { if } t<0 \\
1-e^{-q t} & \text { if } \quad t \geqslant 0
\end{array} \quad(0<q<\infty) .\right.
\end{gathered}
$$

A (standard) substochastic transition matrix is a matrix $\left(p_{i j}\right),(i, j) \in \mathbf{I} \times \mathbf{I}$, of functions on $\mathbf{T}$ satisfying the following conditions:

$$
\begin{gathered}
p_{i j}(t) \geqslant 0, \\
\sum_{j} p_{i j}(s) p_{j k}(t)=p_{i k}(s+t), \\
\lim _{t \downarrow 0} p_{i j}(t)=\delta_{i j}, \\
\sum_{j} p_{i j}(t) \leqslant 1 .
\end{gathered}
$$

It is called stochastic iff equality holds in (2.4) for every $i$ and $t$, and strictly substochastic otherwise. In the latter case we define

$$
p_{i \theta}(t) \equiv 1-\sum_{j \in \mathbf{I}} p_{i j}(t), \quad p_{\theta i}(t) \equiv 0, \quad p_{\theta \theta}(t) \equiv 1
$$

(1) In honor of Feller. 
and call the new matrix enlarged by the index $\theta$ the stochastic completion of $\left(p_{i j}\right)$. The stochastic completion of a stochastic transition matrix is defined to be itself. Given the substochastic $\Pi=\left(p_{i j}\right)$, we define $\mathbf{I}_{\theta}$ to be $\mathbf{I} \cup\{\theta\}$ or $\mathbf{I}$ according as $\Pi$ is strictly substochastic or stochastic, and define $\prod_{\theta}$ to be its stochastic completion.

It is known that each $p_{i j}$ has a right-hand derivative at zero, to be denoted as follows:

$$
p_{i j}^{\prime}(0)=q_{i j}, \quad-q_{i i}=q_{i}
$$

These numbers satisfy the following relations:

$$
\begin{gathered}
-\infty \leqslant q_{i i} \leqslant 0, \quad 0 \leqslant q_{i j}<\infty, \\
\sum_{j} q_{i j} \leqslant 0 .
\end{gathered}
$$

The state $i$ is called stable or instantaneous according as $q_{i}<\infty$ or $q_{i}=\infty$; and it is absorbing iff $q_{i}=0$. The matrix $\left(q_{i j}\right)$ will be called the initial derivative matrix of $\Pi$ and it is said to be conservative iff equality holds in (2.8) for every $i$.

Associated with any matrix $Q=\left(q_{i j}\right)$ subject to the conditions (2.7) and (2.8) are two systems of Kolmogorov differential equations:

$$
\begin{aligned}
& z_{i j}^{\prime}(t)=\sum_{k} q_{i k} z_{k j}(t), \\
& z_{i j}^{\prime}(t)=\sum_{k} z_{i k}(t) q_{k j} .
\end{aligned}
$$

The minimal solution to both systems, first constructed by Feller, will be denoted by $\Phi=\left(f_{i j}\right)$. It is a substochastic transition matrix whose initial derivative matrix is the given $Q$. It is minimal in this sense: if any substochastic transition matrix $\left(p_{i j}\right)$ has the initial derivative matrix $Q$, then

$$
f_{i j}(t) \leqslant p_{i j}(t)
$$

for every $i, j$ and $t$.

A (temporally) homogeneous Markov chain, or Markov chain with stationary transition probabilities, associated with $\mathbf{I}$ and $\Pi$, is a stochastic process $\left\{x_{t}\right\}, t \in \mathbf{T}$ or $t \in \mathbf{T}^{\mathbf{0}}$, on the probability triple $(\Omega, \mathfrak{F}, \mathbf{P})$, having the following properties:

(i) For each $t$ in $\mathbf{T}$ or $\mathbf{T}^{0}$ respectively, $x_{t}=x(t)$ is a discrete random variable, and the set of all possible values of all $x_{t}$ is $\mathbf{I}_{\theta}$.

(ii) If $t_{1}<\ldots<t_{n}$, and $i_{1}, \ldots, i_{n}$ are elements of $\mathbf{I}_{\theta}$, then

$$
\begin{aligned}
\mathbf{P}\left\{x\left(t_{n+1}\right)\right. & \left.=i_{n+1} \mid x\left(t_{v}\right)=i_{v}, 1 \leqslant \nu \leqslant n\right\} \\
& =\mathbf{P}\left\{x\left(t_{n+1}\right)=i_{n+1} \mid x\left(t_{n}\right)=i_{n}\right\}=p_{i_{n} i_{n+1}}\left(t_{n+1}-t_{n}\right) .
\end{aligned}
$$


A version of the process will be chosen to have the further properties:

(iii) For every $\omega$ in $\Omega$,

$$
x(t, \omega)=\varliminf_{r \downarrow t, r \in \mathbf{R}} x(r, \omega)
$$

for every $t$; in particular, the process is right separable with $\mathbf{R}$.

(iv) As a function of $(t, \omega), x(\cdot, \cdot)$ is measurable with respect to $\mathfrak{B} \times \mathfrak{F}$ where $\mathfrak{B}$ is the usual Borel field on $\mathbf{T}$; namely, the process is Borel measurable.

From now on the process $\left\{x_{t}\right\}$ specified as in the above will be referred to as the given Markov chain and abbreviated as $x$. It is called open iff the parameter set is $\mathbf{T}^{\mathbf{0}}$. The set $\mathbf{I}_{\theta}$ is called its (minimal) state space, each element of it being a state, and the matrix $\prod_{\theta}$ is called its transition matrix. The distribution of $x_{0}$, to be always concentrated on $\mathbf{I}$ rather than $\mathbf{I}_{\theta}$, is called its initial distribution and denoted by $\gamma=\left\{\gamma_{i}\right\}$, where

$$
\gamma_{i}=\mathbf{P}\{x(0)=i\}
$$

When $\gamma_{i}=1$, the resulting $\mathbf{P}$ will be written as $\mathbf{P}_{i}$. Mathematical expectation with respect to $\mathbf{P}$ is denoted by $\mathbf{E}$, and conditional probabilities and expectations are denoted by $\mathbf{P}(\cdot \mid \cdot)$ and $\mathbf{E}(\cdot \mid \cdot)$ in the usual way.

A set like $\{\omega: x(t, \omega)=j\}$ is also written more briefly as $\{x(t)=j\}$. The indicator function for the set $\Lambda$ is defined as follows:

$$
1(\Lambda)=1_{\Lambda}(\omega)=\left\{\begin{array}{lll}
0 & \text { if } & \omega \notin \Lambda \\
1 & \text { if } & \omega \in \Lambda
\end{array}\right.
$$

A property involving $\omega$ which is true for almost every $\omega$ is sometimes stated without the qualification "almost every". This can be achieved by suitably restricting the space $\Omega$ at the outset. The Borel field $\tilde{F}$ is assumed to be complete with respect to $\mathbf{P}$ and any of its subfields is supposed to be augmented, namely it contains all null sets. The smallest augmented Borel field with respect to which every $x_{s}, 0 \leqslant s \leqslant t$, is measurable is denoted by $\mathfrak{F}_{t}$.

A number of basic assumptions regarding $\Pi$ or $x$ will be gradually imposed as we proceed in the paper. They are not repeated in every theorem but any theorem given after certain assumptions have been announced is asserted to be valid under these assumptions (though they may be valid without some of them), unless exceptions are specified.

We now make the following assumption which is to hold throughout this paper. 
Assumpton A. For every $i \in \mathbf{I}$,

(A)

$$
-\infty<q_{i i}<0, \quad \sum_{j} q_{i j}=0
$$

The first part of (A) says that every state (except $\theta$ if present) is stable and not absorbing. The second part of (A) is analytically equivalent to the validity of the first system of equations $\left(\hat{I}_{i j}\right)$ for every $i$ and $j$. Together they imply the following properties of the process $([1 ; \S$ II.19]).

(Almost) every sample function executes an infinite sequence of jumps at the times $0<\tau_{1}<\tau_{2}<\ldots$. Let $\tau_{0}=0$, and

$$
\chi_{n}=x\left(\tau_{n}\right) \quad(n \in \mathbf{N})
$$

then

$$
x(t)=\chi_{n} \quad \text { for } \quad t \in\left[\tau_{n}, \tau_{n+1}\right),
$$

and

$$
\mathbf{P}\left\{\tau_{n+1}-\tau_{n} \leqslant t \mid x\left(\tau_{0}\right), \ldots, x\left(\tau_{n}\right)\right\}=e_{a_{x\left(\tau_{n}\right)}}(t)
$$

Let

$$
r_{i j}=\frac{\left(1-\delta_{i j}\right) q_{i j}}{q_{i}}
$$

and $\mathbf{I}_{0}=\left\{j: \sum_{i} \gamma_{i} r_{i j}>0\right\}$. It is clear that under (A) the matrix $\mathbf{P}=\left(r_{i j}\right)$ is stochastic. The stochastic process $\chi=\left\{\chi_{n}, n \in \mathbf{N}\right)$ is a discrete parameter Markov chain with $\gamma$ as its initial distribution, $\mathbf{I}_{0}$ as its state space, and $P$ restricted to $\mathbf{I}_{0} \times \mathbf{I}_{0}$ as its one-step transition matrix. It is called the jump chain associated with $x$. Let

$$
\tau=\lim _{n \rightarrow \infty} \tau_{n}
$$

then $\tau$ is a random variable which may be infinite with positive probability. It is called the first infinity of $x$.

$$
\text { Define further } \quad \bar{x}(t, \omega)= \begin{cases}x(t, \omega) & \text { for } t \in[0, \tau(\omega)), \\ \theta^{\prime} & \text { for } t \in[\tau(\omega), \infty)\end{cases}
$$

Then the stochastic process $\vec{x}=\{\bar{x}(t), t \in \mathbf{T}\}$ is a homogeneous Markov chain with $\gamma$ as its initial distribution, $\mathbf{I}_{0}$ as its state space, and the stochastic completion of $\left(f_{i j}\right)$ by $\theta^{\prime}$ as its transition matrix. It is called the minimal chain associated with $x$. Finally, let

$$
L_{i}(t) \equiv f_{i 0^{*}}(t) \equiv \mathbf{1}-\sum_{j} f_{i j}(t)
$$

then we have

$$
L_{i}(t)=\mathbf{P}_{i}\{\tau \leqslant t\}
$$


Let us remark that if $Q_{\theta}$ and $\Phi_{\theta}$ are the initial derivative matrix and minimal solution associated with $\Pi_{\theta}$, they are defined on the index set $\mathbf{I}_{\theta}$ but not necessarily the stochastic completion of $Q$ and $\Phi$. Under Assumption A, it is easy to see that we have

$$
\begin{array}{lll}
q_{i \theta}=0, & q_{\theta i}=0, & q_{\theta \theta}=1 ; \\
f_{i \theta} \equiv 0, & f_{\theta i} \equiv 0, & f_{\theta \theta} \equiv 1, \quad L_{\theta} \equiv 0 .
\end{array}
$$

It will be noticed that most formulas involving $\theta$ are either trivial or easily derived from those involving only indices in I. The extra index $\theta$ is introduced in order to employ the established formal language of probability theory which requires a total probability of one, even when we begin with a substochastic matrix.

\section{§ 3. The Boundary}

Given the matrix $P=\left(r_{i j}\right)$ defined in (2.12), we now choose the initial distribution $\gamma$ such that $\gamma(i)>0$ for every $i \in \mathbf{I}$, so that the jump chain $\chi$ has $I$ as its state space. Until Theorem 3.2 such terminology as "almost closed", "invariant" and "recurrent" refers to $\chi$. According to a theorem by Blackwell (see $[1 ; \S 1.17]$ ), the set I can be decomposed as follows:

$$
\mathbf{I}=\bigcup_{a} A^{a}
$$

where the index $a$ ranges over a nonvoid, finite or denumerable set and where each $A^{a}$ is an almost closed set, at most one of which is completely nonatomic while every other one (if any) is atomic. Furthermore if we write

$$
L\left(A^{a}\right) \doteq \lim _{n} \sup \left\{\chi_{n} \in A^{a}\right\} \doteq \lim _{n} \inf \left\{\chi_{n} \in A^{a}\right\},
$$

where " $="$ denotes equality modulo a null set, then we have

$$
\sum_{a} \mathbf{P}\left\{L\left(A^{a}\right)\right\}=1
$$

Without loss of generality we may suppose that the sets $L\left(A^{a}\right)$ are disjoint. The mapping $A \rightarrow L(A)$ is a lattice isomorphism between the Borel field of equivalence classes of almost closed sets and that of equivalence classes of nonnull invariant sets. We recall that two almost closed sets are equivalent iff they differ by a transient set, and two invariant sets are equivalent iff they differ by a null set.

We define for each $a$ :

$$
\tau^{a}=\left\{\begin{array}{lll}
\tau & \text { on } & L\left(A^{a}\right), \\
\infty & \text { on } & \Omega \backslash L\left(A^{a}\right) ;
\end{array}\right.
$$


and

$$
\Delta=\{\tau<\infty\}, \quad \Delta^{a}=\left\{\tau^{a}<\infty\right\} .
$$

It follows that $\tau^{a}(\omega)<\infty$ for at most one value of $a$ on $\Omega$, and for exactly one value of $a$ on $\Delta$. Note that

$$
\Delta^{a}=\Delta \cap L\left(A^{a}\right)
$$

and that $\Delta^{a}$ may be a null set; in such a case certain definitions and propositions below are vacuously true.

We now introduce the boundary for $\chi$. In the state space $I$ let the set of nonrecurrent states be $\mathbf{J}^{\prime}$ and let the distinct classes of recurrent states be $\mathbf{I}_{j}^{\prime \prime}$ where $j$ ranges over a possibly void, finite or denumerable set of indices $\mathbf{J}^{\prime \prime}$. Let $\mathbf{J}=\mathbf{J}^{\prime} \cup \mathbf{J}^{\prime \prime}$; thus $\mathbf{J}$ is obtained from $\mathbf{I}$ by leaving the nonrecurrent states alone and identifying the states in each recurrent class as a new state. The theory of Martin boundary ([5], [8]; see also [4]) as applied to $\chi$ has the following consequences.

There exists a compact metric space $J^{*}$ in which $J$ is dense and each element of $J$ is an isolated point. In other words, $J^{*}$ is a compact metrization of $J$, in which the relative topology of $J$ is its natural discrete topology. The set

$$
\mathbf{B}=\left(\mathbf{J}^{*} \backslash \mathbf{J}\right) \cup \mathbf{J}^{\prime \prime}
$$

is called the exit boundary, $\mathbf{J}^{*} \backslash \mathbf{J}$ the nonrecurrent part and $\mathbf{J}^{\prime \prime}$ the recurrent part. For almost every $\omega$, the sequence of random variables $\left\{\chi_{n}, n \in \mathbf{N}\right\}$ behaves in one of the following two alternative ways:

(i) either $\chi_{n}(\omega)$ converges in the metric of $\mathbf{J}^{*}$ to a point in $\mathbf{J}^{\prime \prime}$; this happens if and only if for some $j$ in $\mathbf{J}^{\prime \prime}$ and some $m$ in $\mathbf{N}$, we have $\chi_{n}(\omega) \in I_{j}^{\prime \prime}$ for all $n \geqslant m$;

(ii) or $\chi_{n}(\omega)$ converges in the metric of $\mathbf{J}^{*}$ to a point in $\mathbf{J}^{*} \backslash \mathbf{J}$; this happens if and only if $\chi_{n}(\omega) \in \mathbf{J}^{\prime}$ for all $n$ in $\mathbf{N}$.

In both cases the limit, which is a random variable taking values in the boundary set $\mathbf{B}$, will be denoted by $\chi_{\infty}(\omega)$. Let $\widetilde{S}$ be the topological Borel field of the metric space $\mathbf{J}^{*}$; the boundary measure $\mu$ is defined as follows:

$$
\mu(C)=\mathbf{P}\left\{\chi_{\infty} \in C\right\} \quad(C \in(\mathbb{S}) .
$$

Clearly we have $\mu\left(\mathbf{J}^{\prime}\right)=0$. For a singleton $\{b\} \subset \mathbf{B}$ we write $\mu(b)$ for $\mu(\{b\})$. A point $b$ in $\mathbf{B}$ such that $\mu(b)>0$ is called an atomic boundary point. Every existing recurrent class forms such a point. The set of atomic boundary points is called the (completely) atomic part of the boundary, the remaining part the (completely) nonatomic part. Either part may be void. 
THEOREM 3.1. There is a one-to-one-to-one correspondence between an atomic invariant set $\Lambda$, an atomic almost closed set $A$, and an atomic boundary point $b$ such that

$$
\Lambda \doteq L(A) \doteq\left\{\chi_{\infty}=b\right\}
$$

This being so, the respective nonatomic parts are in similar correspondence.

Proof. The first correspondence in (3.9) is Blackwell's theorem cited above, and the second one is a simple consequence of a result due to Hunt [8], according to which the Borel field of all invariant sets coincides with the smallest Borel field with respect to which $\chi_{\infty}$ is measurable. The proof is terminated.

To proceed to the corresponding boundary for $x$, the time element will now be introduced. We know [1; Theorem II.19.1] that

$$
\{\tau<\infty\} \doteq\left\{\sum_{n=0}^{\infty} \frac{1}{q_{x_{n}}}<\infty\right\}
$$

making it manifest that the set $\{\tau<\infty\}$ is invariant and so by Hunt's result just quoted, there exists a subset $\mathbf{B}_{0}$ of $\mathbf{B}$, belonging to $\mathfrak{E}$, such that

$$
\{\tau<\infty\} \doteq\left\{\chi_{\infty} \in \mathbf{B}_{0}\right\}
$$

Clearly $\mathbf{B}_{0}$ is a subset of the nonrecurrent part of the boundary; $\mathbf{B}_{0}$ is called the passable part, and $\mathbf{B} \backslash \mathbf{B}_{0}$ the impassable part of the boundary. It is important to remark that while $\mathbf{B}$ depends only on $\left(r_{i j}\right), \mathbf{B}_{0}$ depends on $\left\{q_{i}\right\}$ as well, namely it depends on $\left(q_{i j}\right)$.

For each $s$ in $\mathbf{T}$, let $\tau_{s, 0}(\omega)=s$ and $\chi_{s, 0}(\omega)=x_{s}(\omega)$. Let the successive times of jump of $x(\cdot, \omega)$ after $s$ be $\left\{\tau_{s, n}(\omega), n \in \mathbf{N}\right\}$ and let

$$
\chi_{s, n}(\omega)=x\left(\tau_{s, n}(\omega), \omega\right) \quad(n \in \mathbf{N})
$$

The process $\left\{\chi_{s, n}, n \in \mathbf{N}\right\}$ is called the jump chain starting at time $s$; it has properties similar to $\chi$ which is just the special case where $s=0$. Let

$$
\tau_{s, \infty}=\lim _{n \rightarrow \infty} \tau_{s, n}, \quad \chi_{s, \infty}=\lim _{n \rightarrow \infty} \chi_{s, n}
$$

the latter limit being again in the topology of $J^{*}$. We shall say that after the given time $s$, the boundary is first reached at time $\tau_{s, \infty}$ and at the point $\chi_{s, \infty}$. Given a subinterval $S$ of $\mathrm{T}$, the boundary is reached in $S$ at $b$ iff there is an $s$ in $S$ such that $\tau_{s, \infty} \in S$ and $\chi_{s, \infty}=b$. Note that the state space of $\left\{\chi_{s, n}\right\}$, as well as the corre- 
sponding boundary measure $\mu_{s}(C)=\mathbf{P}\left\{\chi_{s, \infty} \in C\right\}$, may vary with $s$. Since for each given $s$, almost every sample function is constant in an open interval containing $s$, it is sufficient to consider all jump chains starting at rational times. More precisely, for almost all $\omega$, all $\tau_{r, \infty}(\omega)$ and $\chi_{r, \infty}(\omega)$ are well defined simultaneously for all $r$ in $\mathbf{R}$; and for each fixed $s$, we have for almost every $\omega$ (the exceptional null set depending on $s$ ):

$$
\tau_{s, \infty}(\omega)=\lim _{r \rightarrow s} \tau_{r, \infty}(\omega), \quad \chi_{s, \infty}(\omega)=\lim _{r \rightarrow s} \chi_{r, \infty}(\omega)
$$

However, it is false that for almost every $\omega$, there is a first time that the boundary is reached after every (generic, not fixed) $t$. Indeed this may be false for $t$ equal to the first infinity $\tau(\omega)$, and here lies much of the difficulty of the theory.

The boundary concepts given in this section are maximal ones relative to a given matrix $\mathrm{P}$ or $Q$. A smaller boundary can be defined relative, in addition, to a given initial distribution $\gamma$. By choosing an everywhere positive $\gamma$ to begin with we have in effect covered all possible choices of $\gamma$, and so in particular included the boundary of $\left\{\chi_{s, n}\right\}$ for every $s$.

We conclude this section by a description of the set of states from which the nonrecurrent and passable part of boundary can not be reached. A sample path beginning at such a state will either reach the recurrent part of the boundary in finite time or remain indefinitely in some almost closed set, approaching the impassable part of the boundary as times goes to infinity. Let

$$
Z=\left\{i \in \mathbf{I}: L_{i} \equiv 0\right\}
$$

In the following, the notions "stochastically closed" and "recurrence" will be prefixed by $\Pi$ or $\Phi$ according to the transition matrix they refer to.

THEOREM 3.2. The set $Z$ is the set of $i$ such that

$$
\mathbf{P}_{i}\{\tau=\infty\}=1
$$

It is $\prod$-stochastically closed and contains all $\Phi$-recurrent states.

Proof. The first assertion follows at once from (2.16). Next, if $i \in Z$, then by (2.9),

$$
\mathbf{1}=\sum_{j} f_{i j}(t) \leqslant \sum_{j} p_{i j}(t) \leqslant 1
$$

hence $f_{i j} \equiv p_{i j}$. It follows from the definition (2.15) that

$$
L_{i}(s+t)-L_{i}(s)=\sum_{j} f_{i j}(s) L_{j}(t)
$$


hence if $i \in Z$, and $f_{i j}(s)=p_{i j}(s)>0$ for some $s$, then $L_{j} \equiv 0$, proving that $Z$ is $\Pi$ stochastically closed. Furthermore we deduce from (3.17) that for every $m$ and $s$ :

$$
1 \geqslant \sum_{n=0}^{m}\left[L_{i}((n+1) s)-L_{i}(n s)\right] \geqslant \sum_{n=0}^{m} f_{i i}(n s) L_{i}(s)
$$

If $i$ is $\Phi$-recurrent, then $\quad \sum_{n=0}^{\infty} f_{i i}(n s)=\infty$.

It follows from (3.18) and (3.19) that $L_{i}(s)=0$. This being true for any $s$, we conclude that $i \in Z$.

An alternative proof of the last part of the theorem is as follows. By a fundamental result on stable states ([1; Theorem 5.7]), the number of disjoint $i$-intervals for $x(t, \omega)$ is finite in any finite subinterval of $\mathbf{T}$ for almost every $\omega$. Consequently, the total number of disjoint $i$-intervals for the minimal chain is finite whenever $\tau(\omega)<\infty$. On the other hand, if $i$ is $\Phi$-recurrent (and not absorbing), this number must be infinite and so (3.15) must hold, hence $i \in Z$.

Remark. It is possible that $i$ is $\prod$-recurrent and yet $i \notin Z$. We need only take an infinite number of independent copies of an ascending escalator, hitched onto one another (see $[1 ; \S I I .20]$ ). Every state is $\Phi$-nonrecurrent and $\Pi$-recurrent in the resulting chain, and $Z$ is void.

COROLLARY TO THEOREM 3.2. $Z=I$ if and only if the passable part of the boundary is void, or $\Pi=\Phi$.

\section{\$ 4. Fundamental Theorems}

Recalling (3.4) we put $\quad L_{i}^{a}(t)=\mathbf{P}_{i}\left\{\tau^{a} \leqslant t\right\}$;

then $L_{i}^{a}(t)$ is the probability, starting from $i$, that the first infinity is reached no later than at time $t$, and that the jump chain finishes by remaining in the almost closed set $A^{a}$. We have clearly

$$
L_{i}=\sum_{a} L_{i}^{a}
$$

and the analogue of $(3,17)$ holds:

$$
L_{i}^{a}(s+t)-L_{i}^{a}(s)=\sum_{j} f_{i j}(s) L_{j}^{a}(t),(1)
$$

(1) By our convention $i \neq \theta$ and $j \neq \theta$, but we can in virtue of (2.17) extend this and similar formulas to cover the index $\theta$. 
this time from its probabilistic meaning. By a general analytical lemma ([3; Lemma 2]), the equation (4.3) implies that each $L_{i}^{a}$ has a continuous derivative $l_{i}^{a}$ in $\mathbf{T}$ satisfying

$$
l_{i}^{a}(s+t)=\sum_{j} f_{i j}(s) l_{j}^{a}(t)
$$

Furthermore, the Kolmogorov equation $\left(\mathrm{I}_{i \theta^{\prime}}\right)$ for $f_{i \theta^{\prime}}$ (see (2.15)) reduces to

$$
l_{i}^{a}(t)=\sum_{j} q_{i j} L_{j}^{a}(t) .
$$

Since $L_{j}^{a}(0)=0$ it follows that

$$
l_{i}^{a}(0)=0
$$

THEOREM 4.1. We have for every $t: 0<t \leqslant \infty$, with probability one:

$$
\lim _{n \rightarrow \infty} L_{\chi_{n}}^{a}(t)=1\left(\Delta^{a}\right)
$$

Proof. We have $([1 ; \S I I .19])$

$$
\mathbf{E}\left\{\tau-\tau_{n} \mid \chi_{n}\right\}=\sum_{m=n}^{\infty} q_{\chi_{m}}^{-1}
$$

where the series converges on $\Delta$ and diverges on $\Omega \backslash \Delta$. By Lévy's zero-or-one law, we have on $\Delta^{a}$ :

$$
\lim _{n \rightarrow \infty} \mathbf{P}\left\{\tau=\tau^{a} \mid \chi_{n}\right\}=1
$$

and consequently

$$
\lim _{n \rightarrow \infty} \mathbf{E}\left\{\tau^{a}-\tau_{n} \mid \chi_{n}\right\}=0
$$

Since

$$
1-L_{\chi_{n}}^{a}(t)=\mathbf{P}\left\{\tau^{a}-\tau_{n}>t \mid \chi_{n}\right\} \leqslant t^{-1} \mathbf{E}\left\{\tau^{a}-\tau_{n} \mid \chi_{n}\right\}
$$

it follows that the first member in (4.9) converges to zero as $n \rightarrow \infty$. On the other hand, by the same law we have on $\Omega \backslash \Delta^{a}$ :

$$
\lim _{n \rightarrow \infty} \mathbf{P}\left\{\tau^{a}=\infty \mid \chi_{n}\right\}=1
$$

Hence it follows from the first relation in (4.9) that its first member converges to one as $n \rightarrow \infty$. Thus (4.7) is proved for $0<t<\infty$. This trivially implies (4.7) for $t=\infty$ on $\Delta^{a}$, which in turn implies the same on $\Omega \backslash \Delta^{a}$ by (4.2). Theorem 4.1 is proved.

CoROLLARY. For each a such that $\mathbf{P}\left\{\Delta^{a}\right\}>0$, there exists a sequence of states $\left\{i_{n}\right\}$ such that for every $b$ :

$$
\lim _{n \rightarrow \infty} L_{i_{n}}^{b}(\cdot)=\delta^{a b} \varepsilon(\cdot)
$$


In particular if $\quad \hat{l}_{i}^{b}(\lambda)=\int_{0}^{\infty} e^{-\lambda t} d L_{i}^{b}(t)$,

then $\quad \lim _{n \rightarrow \infty} \hat{l}_{i_{n}}^{b}(\lambda)=\delta^{a b}$.

Let $C \in \mathbb{S}$, then $\left\{\chi_{\infty} \in C\right\}$ is an invariant set for $\chi$, hence if we set $U_{i}=\mathbf{P}_{i}\left\{\chi_{\infty} \in C\right\}$, we have

$$
U_{i}=\sum_{j} r_{i j} U_{j} \quad \text { or } \quad \sum_{j} q_{i j} U_{j}=0
$$

Define the function $U_{i}(\cdot)$ on $\mathbf{T}$ as follows:

then we have

$$
U_{i}(t)=U_{i}-\sum_{j} f_{i j}(t) U_{j}
$$

It follows from (4.11) or (4.12) that

$$
U_{i}(s+t)-U_{i}(s)=\sum_{j} f_{i j}(s) U_{j}(t)
$$

hence by the lemma cited after $(4.3)$, each $U_{i}(\cdot)$ has a continuous derivative $u_{i}(\cdot)$ satisfying

$$
u_{i}(s+t)=\sum_{j} f_{i j}(s) u_{j}(t) \quad(s>0, t>0)
$$

If $C$ is a subset of $\mathbf{B} \backslash \mathbf{B}_{0}$, then $U_{i}(\cdot) \equiv 0$ for every $i$ by (4.12), and conversely. Otherwise, $u_{i}(t)>0$ for some $i$ and $t>0$ (see the Appendix for a stronger result). If $C$ is a passable atomic boundary point corresponding to $A^{a}$, then $U_{i}(\cdot)$ reduces to $L_{i}^{a}(\cdot)$.

A set of nonnegative functions $\left\{u_{i}(\cdot)\right\}$ with $u_{i}(0)=0$ for every $i$ and satisfying (4.14) will be called an exit solution for $\Phi$. If the $u_{i}$ 's are nonnegative and satisfy (4.14), and we set

$$
\bar{u}_{i}(t)=u_{i}(t)-\sum_{j} f_{i j}(t) u_{j}(0)
$$

then $\left\{\bar{u}_{i}(\cdot)\right\}$ is an exit solution for $\Phi$.

We now make our second basic assumption:

Assumption B. The passable part of the boundary is nonvoid and completely atomic.

We shall denote these atomic boundary points by $\left\{\infty^{a}, a \in \mathbf{A}\right\}$, where $\mathbf{A}$ is a nonvoid, finite or denumerable index set. We have thus

$$
\{\tau<\infty\}=\bigcup_{a \in \mathbf{A}}\left\{\tau^{a}<\infty\right\}=\bigcup_{a \in \mathbf{A}}\left\{\tau<\infty ; \chi_{\infty}=\infty^{a}\right\} .
$$

THEOREM 4.2. Under Assumption B, every exit solution satisfying

$$
\int_{0}^{\infty} u_{i}(t) d t \leqslant 1
$$


is given by

$$
u_{i}(t)=\sum_{a \in \mathbf{A}} c^{a} l_{i}^{a}(t)
$$

where $0 \leqslant c^{a} \leqslant 1$ for every $a$ in $\mathbf{A}$. Furthermore the representation (4.16) is unique.

Proof. Let $\left\{u_{i}(\cdot)\right\}$ satisfy (4.14) and (4.15). Since the Kolmogorov equation $\left(\mathrm{I}_{i j}\right)$ for $\Phi$ is equivalent to

$$
f_{i j}(s)=\sum_{k \neq i} \int_{0}^{s} e^{-q_{i}(s-v)} q_{i k} f_{k j}(v) d v+\delta_{i j}
$$

we have, upon substitution into (4.14):

$$
u_{i}(s+t)-u_{i}(t)=\sum_{i \neq i} \int_{0}^{s} e^{-q_{i}(s-v)} q_{i k} u_{k}(v+t) d v
$$

It is easy to see that we can let $t \downarrow 0$ termwise under the integral; and integrating the resulting equation over $(0, \infty)$, we obtain

$$
\int_{0}^{\infty} u_{i}(s) d s=\sum_{k+i} q_{i}^{-1} q_{i k} \int_{0}^{\infty} u_{k}(v) d v
$$

Hence if we set $U_{i}=\int_{0}^{\infty} u_{i}(s) d s,\left\{U_{i}\right\}$ is a solution of (4.10) with $0 \leqslant U_{i} \leqslant 1$. By a theorem due to Blackwell $([1 ; \S \mathrm{I} .17])$, to such a solution there corresponds an invariant function $\varphi$ with $0 \leqslant \varphi \leqslant 1$ such that $U_{i}=\mathbf{E}_{i}(\varphi)$. Now decompose $\varphi$ as follows:

$$
\varphi=\sum c^{a} 1\left(\Lambda^{a}\right)+\varphi^{0} \quad\left(0 \leqslant c^{a} \leqslant 1\right),
$$

where $\Lambda^{a}=L\left(A^{a}\right)$ in (3.2), and the sum is over the disjoint atomic invariant sets, $\varphi^{0}$ being the remainder which vanishes on the atomic invariant sets. We have, correspondingly,

$$
U_{i}=\sum c^{a} \mathbf{P}_{i}\left(\Lambda^{a}\right)+\mathbf{E}_{i}\left(\varphi^{0}\right)
$$

and using (4.11), the discussion after (4.14), and Assumption B:

$$
U_{i}(t)=\sum_{a \in \mathbf{A}} c^{a} L_{i}^{a}(t)=\int_{0}^{t} \sum_{a \in \mathbf{A}} c^{a} l_{i}^{a}(s) d s
$$

Upon differentiation we obtain (4.16) a. e. Since $\Sigma_{a} l_{i}^{a}(t)=l_{i}(t)$, the series converges uniformly in every compact interval of $\mathbf{T}$ by Dini's Theorem. It follows that both members of (4.16) are continuous and so the equation holds for every $t$.

Suppose $u_{i}(\cdot)$ has another representation of the form (4.16) with $c^{a}$ replaced by $d^{a}$; it follows that 


$$
\sum_{a \in \mathbf{A}} c^{a} L_{i}^{a}(t)=\sum_{a \in \mathbf{A}} d^{a} L_{i}^{a}(t)
$$

Applying the Corollary to Theorem 4.1, we have $c^{a}=d^{a}$. Thus the representation is unique and Theorem 4.2 is proved.

By the same argument, we see that the set of exit solutions $\left\{l_{i}^{a}(\cdot)\right\}, a \in \mathbf{A}$, is a linearly independent set. The conclusion of Theorem 4.2 may be expressed by saying that this set is the extreme base of the space of exit solutions.

From now on an unspecified super-index $a$ or $b$ denotes an element of $\mathbf{A}$ and an unspecified sum over it extends over $\mathbf{A}$.

For terminology relevant to optionality see $[1 ; \S I I .8-9]$. In particular, if $\tau$ is optional. $\mathfrak{F}_{\tau}$ and $\mathfrak{F}_{\tau}^{\prime}$ denote respectively the pre- $\tau$ and post- $\tau$ fields.

Lем МА. Each $\tau^{a}$ is an optional random variable.

Proof. For each $n \in \mathbf{N}, \tau_{n}$ is optional, as can be seen by induction on $n$. Next, let $\tau_{n, m}=\left[m \tau_{n}+1\right] / m$ for every positive integer $m$. Then $\tau_{n, m}$ is rationally valued and optional, and $\tau_{n, m}<t$ for all sufficiently large $m$ on the set $\{\tau<t\}$. These facts imply that $x\left(\tau_{n, m}\right)$ is measurable with respect to the pre- $\tau$ field $\mathfrak{F}_{\tau}$ for large $m$. Since almost every sample function is constant in a right-hand neighborhood of every $\tau_{n}$, by the basic property of a stable state, we have

$$
\lim _{m \rightarrow \infty} x\left(\tau_{n, m}\right)=x\left(\tau_{n}\right)
$$

with probability one by the specification (iii) in $\S 2$ of $x$. Hence every $x\left(\tau_{n}\right)$ is measurable with respect to $\mathfrak{F}_{\boldsymbol{r}}$. Now

$$
\left\{\tau^{a}<t\right\}=\bigcup_{m=1}^{\infty} \bigcap_{n=m}^{\infty}\left\{\tau_{n}<t ; x\left(\tau_{n}\right) \in A^{a}\right\} \in \tilde{F}_{t} .
$$

The lemma is proved.

Under Assumption $\mathbf{B}$, if $\gamma$ is positive everywhere as in $\S 3$, then $\mathbf{P}\left(\Delta^{a}\right)>0$ for every $a$ in $\mathbf{A}$. For an arbitrary $\gamma$, some $\mathbf{P}\left(\Delta^{a}\right)$ may be zero. In what follows, we shall tacitly suppose that $\mathbf{P}\left(\Delta^{a}\right)>0$ in a discussion involving $\Delta^{a}$.

The post $-\tau^{a}$ process $x^{a}=\left\{x_{t}^{a}, t \in \mathbf{T}^{\mathbf{0}}\right\}$ on $\Delta^{a}$ is defined as follows:

$$
x_{t}^{a}=x^{a}(t)=x\left(\tau^{a}+t\right) .
$$

By the strong Markov property as discussed in [1; $\S$ II. 9], $x^{a}$ is an open, homogeneous Markov chain on the probability triple $\left(\Delta^{a}, \mathfrak{F}^{a}, \mathbf{P}^{a}\right)$, where $\mathfrak{F}^{a}=\mathfrak{F} \cap \Delta^{a}, \mathbf{P}^{a}(\cdot)=\mathbf{P}\left(\cdot \mid \Delta^{a}\right)$, with a certain subset $\mathbf{I}^{a}$ of $\mathbf{I}_{\theta}$ as its state space and with the restriction of $\prod_{\theta}$ to $\mathbf{I}^{a} \times \mathbf{I}^{a}$ as its transition matrix. Properties corresponding to (iii) and (iv) in $\S 2$ also hold. 
The process $x^{a}$ can be extended to the parameter set $\mathbf{T}$ on $\Delta^{a}$ if and only if $x^{a}(0)=$ $x\left(\tau^{a}\right) \in \mathbf{I}_{\theta}$ almost everywhere on $\Delta^{a}$.

The next few theorems and their corollaries form the probabilistic basis of the present investigation.

THEOREM 4.3. For each $a$ in $\mathbf{A}$, each $j$ in $\mathbf{I}_{\theta}$ and each $t>0$, there exists a number $\xi_{j}^{a}(t)$ such that

$$
\lim _{n \rightarrow \infty} \mathbf{P}\left\{\Delta^{a} ; x^{a}(t)=j \mid \chi_{n}\right\}=1\left(\Delta^{a}\right) \xi_{j}^{a}(t)
$$

Proof. For each $n$ and $t$, we have

$$
\tau^{a}+t=\lim _{m \rightarrow \infty}\left\{\tau_{n}+\frac{\left[m\left(\tau^{a}+t-\tau_{n}\right)+1\right]}{m}\right\}
$$

It follows from an argument similar to that in the lemma above that $x\left(\tau^{a}+t\right)$ is measurable with respect to the post- $\tau_{n}$ field $\mathfrak{F}_{\tau_{n}}^{\prime}$ and hence with respect to $\bigcap_{n} \mathfrak{F}_{\tau_{n}}^{\prime}$. This fact and the fact that $\chi$ is Markovian imply that the limit in (4.19) exists by the martingale convergence theorem, and being the limit it is ipso facto an invariant function for $\chi$. On $\Omega \backslash \Delta^{a}$ it is zero by Lévy's zero-or-one law. In general it is constant on each atomic invariant set $\Lambda^{a}=L\left(A^{a}\right)$. This constant is the $\xi_{j}^{a}(t)$ in (4.19). Theorem 4.3 is proved.

Corollary. We have $\quad \mathbf{P}\left\{x^{a}(t)=j \mid \Delta^{a}\right\}=\xi_{j}^{a}(t)$, so that $\left\{\xi_{j}^{a}(t)\right\}$ is the absolute distribution of the post- $\tau^{a}$ process at time $t$.

It follows that

$$
\begin{gathered}
\sum_{i \in \mathbf{I}^{\mathbf{a}}} \xi_{i}^{a}(t)=1 \\
\sum_{i \in \mathbf{I}^{a}} \xi_{i}^{a}(s) p_{i j}(t)=\xi_{j}^{a}(s+t) .
\end{gathered}
$$

In particular by a general analytical lemma ([3; Lemma 1]), each $\xi_{i}^{a}$ is continuous in $\mathbf{T}$.

Define $\mathfrak{F}_{\tau}^{-}=\mathrm{V}_{n} \mathfrak{F}_{\tau_{n}}$. Note that in general $\mathfrak{F}_{\tau}^{-}$is a proper subfield of $\mathfrak{F}_{\tau}$, but $\tau$ is measurable with respect to $\mathfrak{F}_{\tau}$.

THEOREM 4.4. For each $a$ in $\mathbf{A}$, if $\mathrm{M} \in \mathfrak{F}_{\tau}^{-}$and $\mathrm{M}^{\prime} \in \mathfrak{F}_{\tau}^{\prime}$, then

$$
\mathbf{P}\left\{\mathbf{M M}^{\prime} \mid \Delta^{a}\right\}=\mathbf{P}\left\{\mathbf{M} \mid \Delta^{a}\right\} \mathbf{P}\left\{\mathbf{M}^{\prime} \mid \Delta^{a}\right\}
$$

Proof. Let $n \in \mathbf{N}$ and $\mathrm{M} \in \mathfrak{F}_{\tau_{n}}$. If $n<m$, then $\mathfrak{F}_{\tau_{n}} \subset \mathfrak{F}_{\tau_{m}}$ trivially and $\mathfrak{F}_{\tau}^{\prime} \subset \mathfrak{F}_{\tau_{m}}^{\prime}$ by 
the argument at the beginning of the preceding proof. Hence $\mathrm{M} \in \mathfrak{F}_{r_{m}}$ and $\left\{x^{a}(t)=j\right\} \in \widetilde{F}_{\tau_{m}}^{\prime}$ Applying the strong Markov property to $\tau_{m}$, we have [1; Theorem II. 9.3]:

$$
\mathbf{P}\left\{\mathbf{M} ; \Delta^{a} ; x^{a}(t)=j \mid \chi_{m}\right\}=\mathbf{P}\left\{\mathbf{M} \mid \chi_{m}\right\} \mathbf{P}\left\{\Delta^{a} ; x^{a}(t)=j \mid \chi_{m}\right\} .
$$

Integrating over $\Omega$ and letting $m \rightarrow \infty$, we have

$$
\mathbf{P}\left\{\mathbf{M} ; \Delta^{a} ; x^{a}(t)=j\right\}=\int_{\Omega^{m \rightarrow \infty}} \lim _{m} \mathbf{P}\left\{\mathbf{M} \mid \chi_{m}\right\} \mathbf{P}\left\{\Delta^{a} ; x^{a}(t)=j \mid \chi_{m}\right\} d \mathbf{P} .
$$

Now if $\mathfrak{J}$ denotes the invariant field for $\chi$, then $\Delta^{a} \in \mathfrak{J}$ and by the Markovian property of $\chi$ and a simple martingale convergence theorem:

$$
\begin{gathered}
\lim _{m \rightarrow \infty} \mathbf{P}\left\{\mathbf{M} \mid \chi_{m}\right\}=\mathbf{P}\left\{\mathbf{M} \mid \chi_{m}, \chi_{m+1}, \ldots\right\}=\mathbf{P}\{\mathbf{M} \mid \Im\} \\
\int_{\Delta^{a}} \lim _{m \rightarrow \infty} \mathbf{P}\left\{\mathbf{M} \mid \chi_{m}\right\} d \mathbf{P}=\mathbf{P}\left\{\mathbf{M} ; \Delta^{a}\right\} .
\end{gathered}
$$

consequently

Using (4.19) and (4.24) in (4.23), we obtain

$$
\mathbf{P}\left\{\mathbf{M} ; x^{a}(t)=j \mid \Delta^{a}\right\}=\mathbf{P}\left\{\mathbf{M} \mid \Delta^{a}\right\} \xi_{j}^{a}(t) .
$$

Applying the strong Markov property to $\tau^{a}+t$, we obtain furthermore that if $0<t=$ $t_{1}<\cdots<t_{l}$, then

$$
\begin{aligned}
& \mathbf{P}\left\{\mathbf{M} ; x^{a}\left(t_{\nu}\right)=j_{\nu}, \mathbf{1} \leqslant \nu \leqslant l \mid \Delta^{a}\right\} \\
= & \mathbf{P}\left\{\mathbf{M} \mid \Delta^{a}\right\} \xi_{j_{1}}^{a}\left(t_{1}\right) \prod_{\nu=1}^{l-1} p_{i_{\nu} j_{\nu+1}}\left(t_{\nu+1}-t_{\nu}\right) \\
= & \mathbf{P}\left\{\mathbf{M} \mid \Delta^{a}\right\} \mathbf{P}\left\{x^{a}\left(t_{\nu}\right)=j_{v}, 1 \leqslant \nu \leqslant l \mid \Delta^{a}\right\} .
\end{aligned}
$$

This being true for arbitrary $t_{v}$ 's and $j_{v}$ 's we conclude that (4.22) holds for every $\mathrm{M} \in \mathfrak{F}_{\tau_{n}}$ and $\mathrm{M}^{\prime} \in \mathfrak{F}_{\tau}^{\prime}$; since $n$ is arbitrary it holds also for every $\mathrm{M} \in \mathrm{V}_{n} \mathfrak{F}_{\tau_{n}}=\mathfrak{F}_{\tau}^{-}$. Theorem 4.4 is proved.

THEOREM 4.5. For almost every $\omega$ in $\Delta^{a}, t_{n}(\omega) \rightarrow t$ implies

$$
\lim _{n \rightarrow \infty} p_{x_{r, n}(\omega), j}\left(t_{n}(\omega)\right)=\xi_{j}^{a}(t)
$$

for every $r$ in $\mathbf{R}$ and $j$ in $\mathbf{I}_{\theta}$.

Proof. We first prove (4.25) with $r=0$ and all $t_{n}(\omega)$ equal to a fixed $t$, and with the exceptional null set possibly varying with $t$. Given $\varepsilon>0$, there exist $\Gamma$ and $m$ such 
that $\Gamma \subset \Delta^{a}$ and $\mathbf{P}\left(\Delta^{a} \backslash \Gamma\right)<\varepsilon$, and such that if $\omega \in \Gamma$ and $n>m$, we have

$$
\tau^{a}(\omega)-\tau_{n}(\omega)<\varepsilon
$$

The basic property of the stable state $j$ for the post- $\tau_{n}$ process implies that on $\Gamma$ :

$$
\mathbf{P}\left\{\Delta^{a} ; x^{a}(t)=j \mid \chi_{n}\right\} \geqslant \mathbf{P}\left\{x\left(\tau_{n}+t\right)=j \mid \chi_{n}\right\} e^{-q_{j} \varepsilon}=p_{x_{n^{*}}}(t) e^{-q_{j} \varepsilon} .
$$

Letting $n \rightarrow \infty$, then $\varepsilon \rightarrow 0$, we obtain from Theorem 4.3:

$$
\xi_{j}^{a}(t) \geqslant \varlimsup_{n \rightarrow \infty} p_{x_{n}, j}(t)
$$

almost everywhere on $\Delta^{a}$. Similarly we have if $\varepsilon<t$,

$$
\mathbf{P}\left\{\Delta^{a} ; x^{a}(t-\varepsilon)=j \mid \chi_{n}\right\} e^{-a_{j} \varepsilon} \leqslant \mathbf{P}\left\{x\left(\tau_{n}+t\right)=j \mid \chi_{n}\right\}=p_{x_{n, j}}(t)
$$

Passing to limits as before, we obtain

$$
\xi_{j}^{a}(t) \leqslant \lim _{n \rightarrow \infty} p_{\chi_{n}, j}(t)
$$

almost everywhere on $\Delta^{a}$. Thus (4.25) is true in this case. Applying this a doubly denumerable number of times, we infer that for almost every $\omega$ in $\Delta^{a}$, and for a fixed sequence $\left\{r_{m}\right\}$ converging to zero,

$$
\lim _{n \rightarrow \infty} p_{z_{r, n}(\omega), j}\left(r_{m}\right)=\xi_{j}^{a}\left(r_{m}\right)
$$

for every $r, j$ and $m$. For any $\omega$ for which (4.27) holds, and for which furthermore $\chi_{r, n}(\omega) \in \mathbf{I}_{\theta}$ for every $r$ and $n$, we now show that the stronger (4.25) also holds, as follows. Let $\chi_{r, n}(\omega)=i_{n}, t_{n}(\omega)=t_{n}$. For any $t>0$, there exists $m$ such that $t>r_{m}$; hence $t_{n}>r_{m}$ for all sufficiently large $n$. We have by Fatou's lemma and (4.21):

$$
\begin{aligned}
\varliminf_{n \rightarrow \infty} p_{i_{n}, j}\left(t_{n}\right) & \geqslant \sum_{i}{\underset{n \rightarrow \infty}{\lim }}_{n \rightarrow \infty} p_{i_{n}, i}\left(r_{m}\right) p_{i j}\left(t_{n}-r_{m}\right) \\
& =\sum_{i} \xi_{i}^{a}\left(r_{m}\right) p_{i j}\left(t-r_{m}\right)=\xi_{j}^{a}(t) .
\end{aligned}
$$

Consequently by $(4.20)$

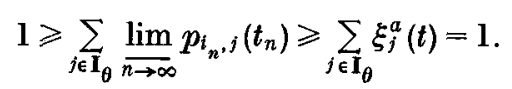

Hence equality holds in (4.28), and this remains true if $\left\{i_{n}\right\}$ is replaced by a subsequence. Therefore the $\lim$ in (4.28) may be replaced by lim and Theorem 4.5 is proved.

4-632932 Acta mathematica. 110. Imprimé le 14 octobre 1963. 
As a consequence of Theorem 4.5, we shall prove a property of sample functions regarding the time set on which the boundary is reached. The corresponding property [1; Theorem II. 6.1], first proved by Doob for an ordinary (vs. a fictitious) state, is a major result in the theory of Markov chains. Two passable atomic boundary points $\infty^{a}$ and $\infty^{b}, a \neq b$, are said to be indistinguishable iff the corresponding post- $\tau^{a}$ and post- $\tau^{b}$ processes have the same finite-dimensional distributions; otherwise they are distinguishable. If $\infty^{a}$ and $\infty^{b}$ are indistinguishable, they can be "merged" as follows. Define

$$
\begin{array}{rlrl}
\left\{\infty^{a \cup b}\right\} & =\left\{\infty^{a}\right\} \cup\left\{\infty^{b}\right\} ; & \Delta^{a \cup b} & =\Delta^{a} \cup \Delta^{b} ; \\
\tau^{a \cup b} & =\left\{\begin{array}{llll}
\tau^{a} & \text { on } & \Delta^{a}, \\
\tau^{b}, & \text { on } & \Delta^{b},
\end{array}\right. & x^{a \cup b}(t)=\left\{\begin{array}{lll}
x^{a}(t) & \text { on } & \Delta^{a}, \\
x^{b}(t), & \text { on } & \Delta^{b} ;
\end{array}\right. \\
L_{i}^{a \cup b}(t) & \equiv L_{i}^{a}(t)+L_{i}^{b}(t), & \xi^{a \cup b}(t) \equiv \xi^{a}(t) \equiv \xi^{b}(t) ;
\end{array}
$$

and treat the union of the two atomic boundary points as if they were one.

For each $\omega$, let the set of $t$ for which there is an $s<t$ such that $\tau_{s, \infty}(\omega)=t$ and $\chi_{s, \infty}(\omega)=\infty^{a}$ be denoted by $S_{\infty}(\omega)$. This is the time set on which the sample function $x(\cdot, \omega)$ reaches the boundary at the point $\infty^{a}$. The union of $S_{\infty}(\omega)$ over $a$ in A may be denoted by $S_{\infty}^{-}(\omega)$ and is the time set on which $x(\cdot, \omega)$ reaches the boundary (under Assumptions $A$ and $B$ ). Note that $t \in S_{\infty}^{-}(\omega)$ does not imply $x(t, \omega)=\infty$, according to the specification (iii) in $\S 2$.

THEOREM 4.6. If $\infty^{a}$ and $\infty^{b}$ are distinguishable, then for almost every $\omega$, no $t$ is a left-hand [or right-hand] $\left(^{1}\right)$ limit point of both $S_{\infty}(\omega)$ and $S_{\infty b}(\omega)$.

Proof. Let $\Omega_{0}$ with $\mathbf{P}\left(\Omega_{0}\right)=1$ be so chosen that (i) every stable state is taken in an open set; (ii) for every $j, s$ and $t$ where $s<t$ the martingale

$$
p_{x(r), j}(t-r)
$$

as $r \downarrow s$ or $r \uparrow s, r \in \mathbf{R}$, has a unique limit; (iii) Theorem 4.5 holds for every $a$. The second stipulation is possible by the regularity properties of the sample functions of a martingale (see [1; p. 153]). We now show that if for some $\omega_{0}$ in $\Omega_{0}$, and an $s$ in To, both $S_{\infty^{a}}\left(\omega_{0}\right)$ and $S_{\infty^{b}}\left(\omega_{0}\right)$ intersect $(s, s+\delta)$ for arbitrarily small $\delta$, then $\xi^{a}(\cdot) \equiv$ $\xi^{b}(\cdot)$. A similar proof holds for $(s-\delta, s)$.

By hypothesis, for every $\delta$ there exists an $r$ in $\mathbf{R}$ such that

(1) $t$ is a left-hand or right-hand limit point of $S$ according as $(t, t+\delta) \cap S \neq 0$ or $(t-\delta, t) \cap$ $S \neq 0$ for every $\delta>0$. 


$$
\tau_{r, \infty} \in(s, s+\delta) \text { and } \chi_{r, \infty}=\infty^{\alpha}
$$

Hence by (i) and (iii) above, there exists a sequence $s_{n} \in \mathbf{R}, s_{n} \uparrow \tau_{r, \infty}$ such that if $t>s+\delta$,

$$
\lim _{n \rightarrow \infty} p_{x\left(s_{n}\right), j}\left(t-s_{n}\right)=\xi_{j}^{a}\left(t-\tau_{r, \infty}\right) .
$$

Since $\delta$ is arbitrary and $\xi_{j}^{a}$ is continuous, this implies that there exists a sequence $r_{n} \in \mathbf{R}, r_{n} \downarrow s$ such that if $t>s$,

$$
\lim _{n \rightarrow \infty} p_{x\left(r_{n}\right), j}\left(t-r_{n}\right)=\xi_{j}^{a}(t-s) .
$$

A similar relation holds for another sequence $r_{n}^{\prime} \in \mathbf{R}, r_{n}^{\prime} \downarrow s$, and $\xi_{j}^{b}$ instead of $\xi_{j}^{a}$. Therefore by (ii) above,

$$
\xi_{j}^{a}(t-s)=\xi_{j}^{b}(t-s)
$$

This being true for every $t>s$, we have $\xi_{j}^{a}(\cdot) \equiv \xi_{j}^{b}(\cdot)$, proving the theorem.

Remark. A point of jump $t$ is a right-hand limit point of some $S_{i}(\omega)$ and a left-hand limit point of a distinct $S_{j}(\omega)$. Without assuming that the states are stable, $i$ and $j$ may be instantaneous if the unilateral limits are replaced by lower limits. From this we surmise that the unilaterality stipulation in the theorem is necessary, though we are not giving a specific counterexample, nor one to show that the distinguishability is also necessary.

\section{§ 5. The First Approach}

The starting point of the analysis of probabilities of transition to and from the boundary is the following result.

Theоrem 5.1. Under Assumptions $A$ and $B$, we have

$$
p_{i j}(t)=f_{i j}(t)+\sum_{a} \int_{0}^{t} l_{i}^{a}(s) \xi_{j}^{a}(t-s) d s
$$

Proof. We have

$$
\mathbf{P}_{i}\left\{x_{t}=j\right\}=\mathbf{P}_{i}\left\{\tau>t ; x_{t}=j\right\}+\sum_{a} \mathbf{P}_{i}\left\{\tau^{a} \leqslant t ; x_{t}=j\right\} .
$$

The first term on the right side is, according to (2.14), $\mathbf{P}_{i}\left\{\bar{x}_{t}=j\right\}=f_{i j}(t)$. Next, the conditional independence asserted in Theorem 4.4 implies, by [1; Theorem II. 9.4], that,

$$
\mathbf{P}_{i}\left\{\tau^{a} \leqslant t ; x_{t}=j\right\}=\int_{0}^{t} \xi_{j}^{a}(t-s) d L_{i}^{a}(s)=\int_{0}^{t} l_{i}^{a}(s) \xi_{j}^{a}(t-s) d s .
$$

Substituting into (5.2) we obtain (5.1). Theorem 5.1 is proved.

For any subinterval $(s, t)$ of $\mathrm{T}^{\mathbf{0}}$, we define 


$$
\begin{aligned}
0^{a}(s, t) & =\left\{\omega: x^{a}(\cdot, \omega) \text { does not reach the boundary in }(s, t)\right\} \\
& =\left\{\omega: x^{a}(\cdot, \omega) \text { has only jumps in }(s, t)\right\} .
\end{aligned}
$$

It is immaterial whether the interval $(s, t)$ is open or closed, provided that a null set can be ignored. Now we define for each $t$ :

$$
\delta_{t}^{a}(\omega)=\delta^{a}(t, \omega)=\inf \left\{s: 0 \leqslant s \leqslant t ; \omega \in 0^{a}(s, t)\right\}
$$

It is easy to see that $\delta_{t}^{a}$ is a random variable with a continuous distribution, which will be given shortly. We call $\delta^{a}(t, \omega)$ the last exit from the boundary before time $t$ for the sample function $x^{a}(\cdot, \omega)$. By definition $\delta^{a}(t, \omega)$ either belongs to or is a right-hand limit point of the set

$$
S_{\infty}(\omega)=\bigcup_{a \in \mathbf{A}} S_{\infty}(\omega)
$$

but it may be the left-hand endpoint of a stable interval. Even if $\mathbf{A}$ is finite, $\delta^{a}(t, \omega)$ may be a right-hand limit point of a certain $S_{\infty b}(\omega)$ (see Theorem 4.5), and not belong to $S_{\infty}^{-}(\omega)$.

It follows from the Corollary to Theorem 4.3 that if $0<s<t$, then

$$
\begin{gathered}
\mathbf{P}^{a}\left\{\delta_{t}^{a} \leqslant s ; x_{t}^{a}=j\right\}=\sum_{i \in \mathbf{I}^{a}} \xi_{i}^{a}(s) f_{i j}(t-s) \quad\left(j \in \mathbf{I}^{a}\right) ; \\
\mathbf{P}^{a}\left\{\delta_{t}^{a} \leqslant s\right\}=\sum_{i \in \mathbf{I}^{a}} \xi_{i}^{a}(s)\left[\mathbf{l}-L_{i}(t-s)\right]=\mathbf{1}-\sum_{i \in \mathbf{I}^{a}} \xi_{i}^{a}(s) L_{i}(t-s) .
\end{gathered}
$$

In dealing with $x^{a}$, the appropriate state space is $\mathbf{I}^{a}$ (which may or may not include $\theta$ ) as noted above, but we shall frequently omit it when no confusion can arise. We now define

$$
\begin{gathered}
\zeta_{j}^{a}(t)=\mathbf{P}^{a}\left\{\delta_{t}^{a}=0 ; x_{t}^{a}=j\right\} \\
\varrho^{a}(t)=\mathbf{P}^{a}\left\{\delta_{t}^{a}>0\right\} .
\end{gathered}
$$

THEOREM 5.2. We have

$$
\begin{gathered}
\zeta_{j}^{a}(t)=\lim _{s \downarrow 0} \sum_{i} \xi_{i}^{a}(s) f_{i j}(t-s)=\lim _{s \downarrow 0} \sum_{i} \xi_{i}^{a}(s) f_{i j}(t) ; \\
\varrho^{a}(t)=\lim _{s \downarrow 0} \sum_{i} \xi_{i}^{a}(s) L_{i}(t-s)=\lim _{s \downarrow 0} \sum_{i} \xi_{i}^{a}(s) L_{i}(t) ; \\
\varrho^{a}(t)+\sum_{j} \zeta_{j}^{a}(t)=1 ; \\
\sum_{i} \zeta_{i}^{a}(s) f_{i j}(t)=\zeta_{j}^{a}(s+t) .
\end{gathered}
$$


Proof. The first equations in (5.9) and (5.10) follow upon letting $s \downarrow 0$ in (5.5) and (5.6); indeed in either case there is monotone convergence. The second equations follow from the stochastic continuity of $\delta_{t}^{a}$ and $x_{t}^{a}$. Analytically, the second equation in (5.9), e.g., can be proved by the inequalities, valid for $0<s<t, 0<s<\delta$ :

$$
f_{i j}(t-s) f_{j j}(s) \leqslant f_{i j}(t) \leqslant f_{i j}(t+\delta-s)\left[f_{j j}(\delta-s)\right]^{-1},
$$

and the continuity of $\zeta_{j}^{a}$ to be noted below. Summing (5.7) over $j$ and adding (5.8) we obtain (5.11). Finally, (5.12) is obvious from the meaning of $\zeta_{j}^{a}(t)$ as the probability that $x^{a}(t, \omega)=j$ and $\omega \in 0^{a}(0, t)$. An analytic proof using (5.9) is also immediate if we observe that

$$
\sum_{i} \xi_{i}^{a}(s) f_{i j}(t-s) \leqslant \sum_{i} \xi_{i}^{a}(s) p_{i j}(t-s)=\xi_{j}^{a}(t)
$$

so that the series

$$
\sum_{j}\left\{\sum_{i} \xi_{i}^{a}(s) f_{i j}(t-s)\right\}
$$

in $j$ is uniformly convergent in $s \in(0, t)$.

TheOREM 5.3. For every $a$ and $\boldsymbol{b}$ in $\mathbf{A}$, there exists a nonnegative nondecreasing function $L^{a b} \leqslant 1$ and a sequence $s_{n} \downarrow 0$ such that

$$
L^{a b}(t)=\lim _{n \rightarrow \infty} \sum_{i} \xi_{i}^{a}\left(s_{n}\right) L_{i}^{b}\left(t-s_{n}\right)=\lim _{n \rightarrow \infty} \sum_{i} \xi_{i}^{a}\left(s_{n}\right) L_{i}^{b}(t)
$$

for every $t>0$. The function $L^{a b}$ is absolutely continuous in $\mathrm{T}^{0}$ but may have a jump at zero; its almost everywhere derivative $l^{a b}$ satisfies, for almost every $t$, the equation

$$
l^{a b}(t)=\sum_{j} \zeta_{j}^{a}(s) l_{j}^{b}(t-s) \quad(0<s<t) .
$$

Proof. The set of functions $\sum_{i} \xi_{i}^{a}(s) L_{i}^{b}(t-s)$ of $t$ in $\left(m^{-1}, \infty\right)$ indexed by $s \in\left(0, m^{-1}\right)$ consists of bounded, nondecreasing functions. Hence by Helly's theorem a sequence $\left\{s_{n}\right\}$ exists for which the first relation in (5.14) holds for $t \in\left(m^{-1}, \infty\right)$. Letting $m \rightarrow \infty$ and using the diagonal argument we obtain the first relation in (5.14) as asserted. Now we have by (4.3), if $s<t_{1}<t_{2}$;

$$
\sum_{i} \xi_{i}^{a}(s)\left\{L_{i}^{b}\left(t_{2}-s\right)-L_{i}^{b}\left(t_{1}-s\right)=\right\} \sum_{j}\left\{\sum_{i} \xi_{i}^{a}(s) f_{i j}\left(t_{1}-s\right)\right\} L_{j}^{b}\left(t_{2}-t_{1}\right) .
$$

Letting $s \downarrow 0$ along the sequence $\left\{s_{n}\right\}$ and using (5.9), (5.14) and the remark involving (5.13), we obtain

$$
L^{a b}\left(t_{2}\right)-L^{a b}\left(t_{1}\right)=\sum_{j} \zeta_{j}^{a}\left(t_{1}\right) L_{j}^{b}\left(t_{2}-t_{1}\right)
$$


Consequently $L^{a b}$ is continuous in $\mathrm{T}^{0}$ and the second relation in (5.14) follows from the first as in Theorem 5.2. Furthermore it follows that

$$
L^{a b}\left(t_{2}\right)-L^{a b}\left(t_{1}\right)=\int_{0}^{t_{2}-t_{1}} \sum_{j} \zeta_{j}^{a}\left(t_{1}\right) l_{j}^{b}(u) d u
$$

If $0<r<s$, we have by (5.12) and (4.4):

$$
\begin{aligned}
\sum_{j} \zeta_{j}^{a}(s) l_{j}^{b}(u) & =\sum_{j}\left\{\sum_{i} \zeta_{i}^{a}(r) f_{i j}(s-r)\right\} l_{j}^{b}(u) \\
& =\sum_{i} \zeta_{i}^{a}(r) \sum_{j} f_{i j}(s-r) l_{j}^{b}(u) \\
& =\sum_{i} \zeta_{i}^{a}(r) l_{i}^{b}(u+s-r) .
\end{aligned}
$$

Hence we can define a function $l^{a b}$ by (5.15) and substituting into (5.17) we obtain

$$
L^{a b}\left(t_{2}\right)-L^{a b}\left(t_{1}\right)=\int_{t_{1}}^{t_{2}} l^{a b}(s) d s \quad\left(0<t_{1}<t_{2}<\infty\right) .
$$

COROLLARY, The left member of (5.16) converges as $s \downarrow 0$ to the left member of (5.17).

Proof. This follows since each sequence $\left\{s_{n}\right\}$ converging to zero contains a subsequence along which the left member of (5.16) converges to the unique limit given in (5.19).

Remark. The corollary says that the measures in $t$ corresponding to $\sum_{i} \xi_{i}^{a}(s) L_{i}^{b}(t-s)$ converge on $\mathbf{T}^{0}$ as $s \downarrow 0$. We do not know if they converge on $\mathbf{T}$. More precisely, define $L^{a b}(0)=0$ and let the jump of $L^{a b}$ at zero be denoted by $L^{a b}(0+)$; does

$$
\lim _{t \downarrow 0} \lim _{s \downarrow 0} \sum_{i} \xi_{i}^{a}(s) L_{i}^{b}(t-s)
$$

exist and equal to $L^{a b}(0+)$ ?

ThEOREM 5.4. Under Assumptions $A$ and $B$, we have

$$
\xi_{j}^{a}(t)=\zeta_{j}^{a}(t)+\sum_{b \in \mathrm{A}} \int_{0}^{t} \xi_{j}^{b}(t-s) d L^{a b}(s)
$$

if and only if

$$
\varrho^{a}(\cdot)=\sum_{b} L^{a b}(\cdot)
$$

Proof. Substituting (5.1) into (4.21), we have

$$
\xi_{j}^{a}(s+t)=\sum_{i} \xi_{i}^{a}(s) f_{i j}(t)+\sum_{b \in \mathbf{A}} \int_{0}^{t} \xi_{j}^{b}(t-u) d_{u}\left[\sum_{i} \xi_{i}^{a}(s) L_{i}^{b}(u)\right]
$$


Since $\mathbf{A}$ is denumerable, there exists by the diagonal argument a sequenee $\left\{s_{n}\right\} \downarrow 0$ such that (5.14) holds for every $a$ and $b$. Since each $\xi_{j}^{b}$ is continuous, this implies the convergence of each integral in (5.22) to its corresponding limit. Hence by (5.9) and Fatou's lemma, (5.20) holds with "=" replaced by " $\geqslant "$. Summing over $j$ in $\mathbf{I}^{a}$, we have

$$
1 \geqslant \sum_{j} \zeta_{j}^{a}(t)+\sum_{b \in \mathbf{A}} L^{a b}(t)
$$

Comparing this with (5.11) we see that (5.21) is a necessary and sufficient condition for the equality to hold in (5.20).

CoRollary. A sufficient condition for the validity of (5.20) is that for each a, there is only a finite number of $b$ such that $L^{a b}(\infty)>0$; this is the case if $\mathbf{A}$ is a finite set.

Let the Laplace transforms be defined as follows, $0<\lambda<\infty$ :

$$
\left.\begin{array}{r}
\hat{\xi}_{j}^{a}(\lambda)=\int_{0}^{\infty} e^{-\lambda t} \xi_{j}^{a}(t) d t ; \quad \hat{\zeta}_{j}^{a}(\lambda)=\int_{0}^{\infty} e^{-\lambda t} \zeta_{j}^{a}(t) d t \\
\hat{L}^{a b}(\lambda)=\int_{0}^{\infty} e^{-\lambda t} d L^{a b}(t)=L^{a b}(0+)+\int_{0}^{\infty} e^{-\lambda t} l^{a b}(t) d t .
\end{array}\right\}
$$

The equation (5.20) becomes, omitting the index $j$ :

$$
\hat{\xi}^{a}(\lambda)=\hat{\zeta}^{a}(\lambda)+\sum_{b} \hat{L}^{a b}(\lambda) \hat{\xi}^{b}(\lambda)
$$

or in matrix form on the super-index:

$$
[I-\Lambda(\lambda)] \hat{\xi}(\lambda)=\hat{\zeta}(\lambda)
$$

where $I$ is the identity matrix,

$$
\Lambda(\lambda)=\left(\hat{L}^{a b}(\lambda)\right), \quad(a, b) \in \mathbf{A} \times \mathbf{A},
$$

and $\hat{\xi}(\lambda)$ and $\hat{\zeta}(\lambda)$ are regarded as column vectors with the components indexed by $\mathbf{A}$.

Following an established terminology [1; 1 I. 3], we write $a \sim b$ iff $L^{a b}(\infty)>0$, otherwise $a \sim b$; and we write $a \sim b$ iff $a \sim b$ and $b \sim a$. Note that an equivalent definition is obtained if we use $\hat{L}^{a b}(\lambda)$ for any $\lambda<\infty$ instead of $L^{a b}(\infty)=\hat{L}^{a b}(0)$. The index $a$ is essential iff there exists $b$ such that $a \sim b$ and for each such $b$ we have also $b \sim a$; otherwise it is inessential. The relation $\sim$ among essential indices is an equivalence relation by means of which they are partitioned into essential classes $C_{1}, C_{2}, \ldots$ The set of inessential indices will be denoted by $C_{0}$. 
THEOREM 5.5 Suppose that $\mathrm{A}$ is a finite set. One of the following alternatives must occur:

(i) There exists an essential class $C$ of indices such that

$$
\sum_{b \in C} L^{a b}(0+)=1 \quad(a \in C)
$$

In this case if $a \in C$ and $b \in C$, then $\infty^{a}$ and $\infty^{b}$ are indistinguishable. $C$ may be a singleton.

(ii) The matrix $I-\Lambda(\lambda)$ is invertible and

$$
\hat{\xi}(\lambda)=[I-\Lambda(\lambda)]^{-1} \hat{\zeta}(\lambda)=\sum_{n=0}^{\infty} \Lambda^{n}(\lambda) \hat{\zeta}(\lambda)
$$

Proof. Suppose there exists an essential class $C$ such that the restriction of $\Lambda(\lambda)$ to it is stochastic, namely, we have

$$
\sum_{b \in C} \hat{L}^{a b}(\lambda)=1 \quad(a \in C) .
$$

This can happen only if $\hat{L}^{a b}(\lambda)=L^{a b}(0+)$ and (5.26) holds. It follows from this, (5.10) and (5.14) that

$$
\varrho^{a}(t)=\lim _{n \rightarrow \infty} \sum_{i} \xi_{i}^{a}\left(s_{n}\right) \sum_{b \in \mathbf{A}} L_{i}^{b}(t)=\sum_{b \in \mathbf{A}} L^{a b}(0+)=1
$$

consequently $\varrho^{a}(0+)=\lim _{t \downarrow} \varrho^{a}(t)=1$ and so $\zeta_{j}^{a}(t)=0$ for every $j$ and $t$ by (5.11). Thus (5.24) reduces to

$$
\hat{\xi}^{a}(\lambda)=\sum_{b \in C} L^{a b}(0+) \hat{\xi}^{b}(\lambda) \quad(a \in C)
$$

Since $C$ is a finite set, being a subset of $\mathbf{A}$, a well-known result in discrete parameter Markov chains asserts that the only solution $\hat{\xi}(\lambda)$ of such a system of equations is a constant. A simple algebraic proof is also available. Thus for each $\lambda, \hat{\xi}^{a}(\lambda)=$ $\hat{\xi}^{b}(\lambda)$ for every $a$ and $b$ in $C$ and we conclude that $\xi_{j}^{a}(t) \equiv \xi_{j}^{b}(t)$ identically in $j$ and $t$ for $a$ and $b$ in $C$, by the uniqueness of Laplace transforms. $\left.{ }^{(}\right)$

On the other hand, if there does not exist any essential class $C$ with the property (5.28), then it is a consequence of the recurrence properties of discrete parameter Markov chains that the series $\sum_{n=0}^{\infty} \Lambda^{n}(\lambda)$ converges for $0<\lambda<\infty$ and yields the inverse of $I-\Lambda(\lambda)$. Applying it to (5.25) we obtain (5.27), completing the proof of Theorem 5.5.

(1) This result should be compared with Theorem 4.6. 
If indistinguishable boundary points have been merged, then the alternative (i) reduces to

$$
L^{a a}(0+)=1
$$

for a certain merged boundary point. This means $\tau^{a}$ is a left-hand limit point of $S_{\infty^{a}}(\omega)$ almost everywhere on $\Delta^{a}$, and the fictitious state $\infty^{a}$ behaves as an instantaneous state. Analytically, the equation (5.24) reduces to the trivial identity $\hat{\xi}^{a}(\lambda)=$ $\hat{\xi}^{a}(\lambda)$.

Under the alternative (ii), the sample functions can be described as follows. For almost every $\omega, x(\cdot, \omega)$ reaches the passable part of the boundary in a sequence of times

$$
\tau(0)<\tau(1)<\tau(2)<\cdots\left({ }^{1}\right),
$$

where $\tau(0)=\tau$ is the first infinity in our previous notation and if $\tau(n)=\infty$ then $\tau(n+1)=\tau(n+2)=\cdots=\infty$. We define

$$
\begin{array}{lll}
y(n)=\lim _{t+\tau(n)} x(t) & \text { if } & \tau(n)<\infty, \\
y(n)=\theta^{\prime \prime} & \text { if } & \tau(n)=\infty ;
\end{array}
$$

where the limit is taken in the metric topology of $\mathrm{J}^{*}$ so that $y(n) \in \mathbf{B}_{0}$. If we write simply " $a$ " for " $\infty^{a}$ ", the process $\{y(n), n \in \mathbf{N}\}$ is a discrete parameter homogeneous Markov chain with $\mathbf{A}_{\theta}$ as its state space, and the stochastic completion (by $\theta^{\prime \prime}$ ) of $\left(L^{a b}(\infty)\right),(a, b) \in \mathbf{A} \times \mathbf{A}$, as its one-step transition matrix. Furthermore we have

$$
\mathbf{P}\{\tau(n+1)-\tau(n) \leqslant t ; y(n+1)=b \mid y(n)=a\}=L^{a b}(t) \text {. }
$$

If we define

$$
z(t-\tau(0))=y(n) \quad \text { for } \quad \tau(n) \leqslant t<\tau(n+1),
$$

the process $\{z(t), t \in \mathbf{T}\}$ is a so-called semi-Markovian process. Finally if we set

$$
v(t)=n \quad \text { for } \quad \tau(n) \leqslant t<\tau(n+1),
$$

then for any $t$ and $\Lambda \in \mathfrak{F}_{\tau(\nu(t))}$,

$$
\mathbf{P}\{x(t)=j \mid \Lambda ; y(\nu(t))=a\}=\zeta_{j}^{a}(t-\tau(\nu(t)) .
$$

Perhaps it is better to describe the above situation in somewhat less precise but more intelligible terms as follows. The sample function of the Markov chain $x$ is

(1) $\tau(n)$ is not the previous $\tau_{n}$. 
composed of a sequence of "waves" going from a passable atomic boundary point to another (not necessarily distinct one). The transition of these boundary points follows that of an imbedded Markov chain with $\left(L^{a b}(\infty)\right)$ as one-step transition matrix. The length of each wave joining $\infty^{a}$ to $\infty^{b}$ has the distribution $L^{a b}(\cdot)$, independently of any occurrence outside this wave. Within each wave the sample function has only jumps, and consequently the transition of $x$ there is by means of $\Phi=\left(f_{i j}\right)$. If a wave begins at $\infty^{a}$, then at $t$ units of time later $x$ is in the state $j$ with probability $\zeta_{j}^{a}(t)$. A sample function may have a finite number of waves before reaching a point on the recurrent part of the boundary and remaining there ever after; or it may have a final wave extending to infinity while approaching the impassable part of the boundary; or it may have an infinite sequence of waves going to infinity. Under the hypothesis of Theorem 5.5, in the case (ii), these waves cannot accumulate in the finite.

\section{§ 6. The Second Approach}

Let $I^{\prime}$ be the set of $\Pi$-nonrecurrent states in $I_{\theta}$; note that $\theta$, if present, is recurrent.

For each $a$ in $\mathbf{A}$ and $j$ in $\mathbf{I}_{\theta}$, we set

$$
g_{j}^{a}=\int_{0}^{\infty} \xi_{j}^{a}(t) d t
$$

THEOREM 6.1. The set of $j$ for which $g_{j}^{a}>0$ is the state space $\mathbf{I}^{a}$ of the post- $\tau^{a}$ process. If $j \in \mathbf{I}^{a}$, then $g_{j}^{a}<\infty$ or $g_{j}^{a}=\infty$ according as $j \in \mathbf{I}^{\prime}$ or $j \notin \mathbf{I}^{\prime}$.

Proof. The first assertion follows from the fact that $I^{a}$ is the set of $j$ for which $\xi_{j}^{a}(t)>0$ for some $j$ and $t$, and the continuity of $\xi_{j}^{a}$. To prove the second assertion, we observe that if $j \in \mathbf{I}^{\prime}$, then $\int_{0}^{\infty} p_{i j}(t) d t<\infty$. We have by $(5.1)$

$$
p_{i j}(t) \geqslant \int_{0}^{t} \xi_{j}^{a}(t-s) d L_{i}^{a}(s)
$$

consequently

$$
\int_{0}^{\infty} p_{i j}(t) d t \geqslant L_{i}^{a}(\infty) g_{j}^{a}
$$

There exists an $i$ in I such that $L_{i}^{a}(\infty)>0$; hence $g_{j}^{a}<\infty$. An alternative proof of this is as follows. If $S_{j}=S_{j}(\omega)=\{t: x(t, \omega)=j\}$ and $\mu$ is the Lebesgue measure on T, then we have 


$$
g_{j}^{a}=\mathbf{E}\left\{\mu\left[S_{j} \cap\left(\tau^{a}, \infty\right)\right]\right\} \leqslant \mathbf{E}\left\{\mu\left[S_{j}\right]\right\}<\infty
$$

by [1; Theorem II. 10.4].

On the other hand, if $j \notin \mathbf{I}^{\prime}$ then $\int_{0}^{\infty} p_{j j}(t) d t=\infty$. We have by $(4.21)$, for any $s$ and $t$ :

$$
\xi_{j}^{a}(s+t) \geqslant \xi_{j}^{a}(s) p_{j j}(t)
$$

It follows that for any $u$ :

$$
g_{j}^{a} \geqslant \int_{0}^{u} \xi_{j}^{a}(s+t) d t \geqslant \xi_{j}^{a}(s) \int_{0}^{u} p_{j j}(t) d t
$$

There exists $s$ such that $\xi_{j}^{a}(s)>0$; hence we obtain $g_{j}^{a}=\infty$ by letting $u \rightarrow \infty$.

AssuMPTION C. $\mathbf{I}=\mathbf{I}$; namely there is no $\Pi$-recurrent state except $\theta$ if present.

It is not true that Assumption $\mathrm{C}$ can be made without loss of generality, even if we are only interested in the nonrecurrent part of the boundary. This is because a $\prod$-recurrent state need not be $\Phi$-recurrent; see Theorem 3.2 and the Remark after it. In particular the Doob type of construction (see [1; Theorem II. 19.4]) leads to $\Pi$ recurrent states if $L_{i}(\infty)=1$ for every $i$.

From now on in this section an unspecified index $i, j$ or $k$ is an element of $\mathbf{I}^{\prime}$, and an unspecified sum over it is extended to $\mathbf{I}^{\prime}$.

$$
\text { For } j \in \mathbf{I}^{\prime} \text {, we set } \quad \mathbf{H}_{j}^{a}(t)=g_{j}^{a}-\sum_{i} g_{i}^{a} f_{i j}(t) \text {. }
$$

This is the "dual" of (4.11).

THEOREM 6.2. We have if $j \in \mathbf{I}^{a}$ :

$$
\begin{gathered}
\mathbf{H}_{j}^{a}>0, \quad \mathbf{H}_{j}^{a} \uparrow, \quad \mathbf{H}_{j}^{a}(0)=0 ; \\
\mathrm{H}_{j}^{a}(s+t)-\mathrm{H}_{j}^{a}(t)=\sum_{i} \mathbf{H}_{i}^{a}(s) f_{i j}(t) .
\end{gathered}
$$

$\mathrm{H}_{j}^{a}$ has a continuous positive derivative $\eta_{j}^{a}$ in $\mathbf{T}$ satisfying

$$
\eta_{j}^{a}(s+t)=\sum_{i} \eta_{i}^{a}(s) f_{i j}(t) .
$$

Proof. We have

$$
\begin{aligned}
\sum_{i} g_{i}^{a} f_{i j}(t) & =\sum_{i} \int_{0}^{\infty} \xi_{i}^{a}(s) f_{i j}(t) d s \leqslant \sum_{i} \int_{0}^{\infty} \xi_{i}^{a}(s) p_{i j}(t) d s \\
& =\int_{0}^{\infty} \xi_{j}^{a}(s+t) d s=g_{j}^{a}-\int_{0}^{t} \xi_{j}^{a}(s) d s \leqslant g_{j}^{a}
\end{aligned}
$$


By the Appendix the second inequality above is strict, hence $\mathrm{H}_{j}^{a}>0$. Next we have

$$
\sum_{i} \mathrm{H}_{i}^{a}(s) f_{i j}(t)=\sum_{i}\left\{g_{i}^{a}-\sum_{k} g_{k}^{a} f_{k i}(s)\right\} f_{i j}(t)=\sum_{i} g_{i}^{a} f_{i j}(t)-\sum_{k} g_{k}^{a} f_{k j}(s+t)=\mathbf{H}_{j}^{a}(s+t)-\mathbf{H}_{j}^{a}(t)
$$

hence $\mathrm{H}_{j}^{a} \uparrow$. The continuous differentiability of $\mathrm{H}_{j}^{a}$ together with (6.5) follows from the equation (6.4) by a general lemma already cited under (4.3) in its dual form. Finally, $\eta_{j}^{a}>0$ by the Appendix.

For every $a$ and $b$ in $\mathbf{A}$, we set

$$
\sigma^{a b}(t)=\sum_{i} g_{i}^{a} l_{i}^{b}(t)
$$

It will follow from the proof below that the series in (6.6) converges for every $t>0$, and is a nonincreasing function of $t$.

The next theorem is fundamental; it takes the place of Theorem 5.4 in the new approach.

Theorem 6.3. Under Assumptions $A, B$, and $C$, we have

$$
\int_{0}^{t} \eta_{j}^{a}(s) d s=\int_{0}^{t} \xi_{j}^{a}(s) d s+\sum_{b \in \mathbf{A}} \int_{0}^{t} \sigma^{a b}(s) \xi_{j}^{b}(t-s) d s
$$

Proof. Let us rewrite (5.22) as

$$
\xi_{j}^{a}(s+t)=\sum_{i} \xi_{i}^{a}(s) f_{i j}(t)+\sum_{b} \int_{0}^{t}\left[\sum_{i} \xi_{i}^{a}(s) l_{i}^{b}(u)\right] \xi_{j}^{b}(t-u) d u .
$$

For each $b$, there exist $j$ and $t_{0}$ such that $\xi_{j}^{b}\left(t_{0}\right)>0$, and this implies $\xi_{j}^{b}(t)>0$ for all $t>t_{0}$ by (4.21) (for a stronger result see the Appendix). It follows from this that the series in square brackets in (6.8) converges for each fixed $s$ and almost every $u$. Furthermore we can integrate to obtain

$$
\infty>\int_{0}^{\infty} \xi_{j}^{a}(s+t) d s=\sum_{i} g_{i}^{a} f_{i j}(t)+\sum_{b} \int_{0}^{t} \sigma^{a b}(u) \xi_{j}^{b}(t-u) d u,
$$

where $\sigma^{a b}$ is defined by (6.6) and the series there converges for almost every $t$. If it converges for $t$ and $t<u$, then by (4.4),

$$
\sum_{i} g_{i}^{a} l_{i}^{b}(u)=\sum_{j}\left[\sum_{i} g_{i}^{a} f_{i j}(u-t)\right] l_{j}^{b}(t) \leqslant \sum_{j} g_{j}^{a} l_{j}^{b}(t)
$$

Hence the series in (6.6) converges for every $t>0$. Finally, since 


$$
\begin{aligned}
\int_{0}^{\infty} \xi_{j}^{a}(s+t) d s-\sum_{i} g_{i}^{a} f_{i j}(t) & =g_{j}^{a}-\int_{0}^{t} \xi_{j}^{a}(s) d s-\sum_{i} g_{i}^{a} f_{i j}(t) \\
& =\mathrm{H}_{j}^{a}(t)-\int_{0}^{t} \xi_{j}^{a}(s) d s \\
& =\int_{0}^{t} \eta_{j}^{a}(s) d s-\int_{0}^{t} \xi_{j}^{a}(s) d s
\end{aligned}
$$

by Theorem $6.2,(6.9)$ is equivalent to $(6.7)$.

Corollary 1. $\mathrm{H}_{j}^{a}(\infty)=g_{j}^{a}$, viz.

$$
\int_{0}^{\infty} \xi_{j}^{a}(s) d s=\int_{0}^{\infty} \eta_{j}^{a}(s) d s .
$$

Proof. We have by (6.7) and (6.2)

$$
\mathrm{H}_{j}^{a}(\infty) \geqslant \int_{0}^{\infty} \xi_{j}^{a}(s) d s=g_{j}^{a} \geqslant \mathrm{H}_{j}^{a}(t)
$$

Corollary 1 follows upon letting $t \rightarrow \infty$.

COROLLARY 2. $\sigma^{a b}$ is summable over every finite interval.

Proof. This follows from (6.7) and the fact, already used in the proof of the theorem, that for each $b$ there exist $j$ and $t_{0}$ such that $\xi_{j}^{b}(t)>0$ for $t>t_{0}$, and consequently $\xi_{j}^{b}$ is bounded away from zero in $\left(t_{0}, t_{1}\right)$ for every $t_{1}>t_{0}$, since it is continuous.

THEOREM 6.4. For almost every $t$, the series

$$
\sum_{i} \eta_{i}^{a}(s) l_{i}^{b}(t-s)
$$

converges for $0<s<t$ and defines a function $\theta^{a b}(t)$ which does not depend on $s$. We have

$$
\sigma^{a b}(t)=\int_{t}^{\infty} \theta^{a b}(s) d s=\sum_{i} \eta_{i}^{a}(t) L_{i}^{b}(\infty) ;
$$

in particular, $\sigma^{a b}$ is continuous in $T^{0}$.

Proof. If the series in (6.10) converges, then the sum does not depend on $s$ for $s$ in $(0, t)$ by exactly the same calculation as given in (5.18). Now by (6.6) and Corollary 1 to Theorem 6.3 , we have

$$
\sigma^{a b}(t)=\sum_{i} \int_{0}^{\infty} \eta_{i}^{a}(s) l_{i}^{b}(t) d s=\int_{0}^{\infty} \theta^{a b}(s+t) d s
$$


for almost every $t$, using Fubini's theorem on product measures; similarly

$$
\sigma^{a b}(t)=\int_{0}^{\infty} \sum_{i} \eta_{i}^{a}(t) l_{i}^{b}(s) d s=\sum_{i} \eta_{i}^{a}(t) L_{i}^{b}(\infty)
$$

Since both the extreme members of $(6.12)$ are nonincreasing and the one on the right is continuous, (6.12) must hold for every $t>0$. Now the first member of (6.13) is nonincreasing and continuous, while the last one is easily seen to be nonincreasing, hence (6.13) must hold for every $t$. Theorem 6.4 is proved.

To proceed further we need an essential strengthening of Assumption B, already imposed in Theorem 5.5.

Assumption $\mathbf{B}^{\prime}$. A is a finite set.

Let us put

$$
\begin{aligned}
& \sigma^{a}(t) \equiv \sum_{b \in \mathbf{A}} \sigma^{a b}(t) \\
& \eta_{*}^{a}(t) \equiv \sum_{i} \eta_{i}^{a}(t) .
\end{aligned}
$$

THEOREM 6.5. Under Assumption $B^{\prime}$ the function $\eta_{*}^{a}$ is finite, nonincreasing and continuous in $\mathbf{T}^{0}$, and summable in every finite interval. We have

$$
\begin{gathered}
\eta_{*}^{a}(t)-\eta_{*}^{a}(\infty)=\sigma^{a}(t) \\
\eta_{*}^{a}(\infty)=\sum_{i} \eta_{i}^{a}(t)\left[1-L_{i}(\infty)\right] .
\end{gathered}
$$

Proof. Summing (6.7) over $j$ and using Corollary 2 to Theorem 6.3, we have

$$
\sum_{j} \mathrm{H}_{j}^{a}(t) \leqslant t+\sum_{b} \int_{0}^{t} \sigma^{a b}(s) d s<\infty,
$$

since $b$ ranges over a finite set. It follows that the series in (6.15) converges for almost every $t$. If $\eta_{*}^{a}(t)<\infty$ then we have by $(6.5)$, for every $t^{\prime} \geqslant 0$ :

$$
\eta_{*}^{a}(t)=\sum_{i} \eta_{i}^{a}(t)\left\{\sum_{j} f_{i j}\left(t^{\prime}\right)+L_{i}\left(t^{\prime}\right)\right\}=\eta_{*}^{a}\left(t+t^{\prime}\right)+\sum_{i} \eta_{i}^{a}(t) L_{i}\left(t^{\prime}\right)
$$

Hence $\eta_{*}^{a}\left(t+t^{\prime}\right)<\infty$ and we conclude that $\eta_{*}^{a}$ is finite and nonincreasing in $\mathbf{T}^{0}$. Its continuity there also follows from (6.19), since each $L_{i}$ is continuous in $\mathbf{T}$. The summability of $\eta_{*}^{a}$ follows from (6.18). Finally, rewriting (4.3) as

$$
1-L_{i}(s+t)=\sum_{j} f_{i j}(s)\left[1-L_{j}(t)\right]
$$

and letting $t \rightarrow \infty$ we see that 


$$
1-L_{i}(\infty)=\sum_{j} f_{i j}(s)\left[1-L_{j}(\infty)\right]
$$

On the other hand, summing $(6.13)$ over $b$ we have

$$
\sigma^{a}(t)=\sum_{i} \eta_{i}^{a}(t) L_{i}(\infty)
$$

It follows from $(6.20)$ and $(6.21)$ that

$$
\begin{aligned}
\eta_{*}^{a}(t)-\sigma^{a}(t) & =\sum_{j} \eta_{j}^{a}(t)\left[1-L_{j}(\infty)\right] \\
& =\sum_{j} \sum_{i} \eta_{i}^{a}(s) f_{i j}(t-s)\left[1-L_{j}(\infty)\right] \\
& =\sum_{i} \eta_{i}^{a}(s)\left[1-L_{i}(\infty)\right]=\eta_{*}^{a}(s)-\sigma^{a}(s)
\end{aligned}
$$

Thus $\eta_{*}^{a}(t)-\sigma^{a}(t)$ is a constant which must be $\eta_{*}^{a}(\infty)$ since $\sigma^{a}(\infty)=0$.

Corollary. $\eta_{*}^{a}(\infty) \leqslant 1$.

Proof. Divide (6.18) by $t$ and let $t \rightarrow \infty$.

The next two theorems are valid under Assumptions $A, B$ and $C$ (without $B^{\prime}$ ). Remember that $j \neq \theta$ below.

THEOREM 6.7. $\eta_{j}^{a}$ is absolutely continuous and

$$
\eta_{j}^{a}(t)=-\sum_{i} g_{i}^{a} f_{i j}^{\prime}(t)
$$

where the series converges absolutely for $t \geqslant 0$; in particular

$$
\eta_{j}^{a}(0)=-\sum_{i} g_{i}^{a} q_{i j}
$$

We have for almost every $t$ :

$$
\frac{d}{d t} \eta_{j}^{a}(t)=\sum_{i} \eta_{i}^{a}(t) q_{i j}
$$

Proof. We have

or

$$
\begin{gathered}
0 \leqslant \frac{\mathrm{H}_{j}^{a}(t)}{t}=\sum_{i} g_{i}^{a} \frac{\left[\delta_{i j}-f_{i j}(t)\right]}{t} \\
\sum_{i \neq j} g_{i}^{a} \frac{f_{i j}(t)}{t} \leqslant g_{j}^{a} \frac{1-f_{j j}(t)}{t} .
\end{gathered}
$$

Letting $t \downarrow 0$ we have by Fatou's lemma

$$
\sum_{i \neq j} g_{i}^{a} q_{i j} \leqslant g_{j}^{a} q_{j}
$$


Next, we have, using the second system of equations (II) in $\S 2$ for $\Phi$ :

by $(6.26)$. It follows that

$$
\begin{aligned}
\sum_{i} g_{i}^{a}\left|f_{i j}^{\prime}(t)\right| & \leqslant \sum_{i} g_{i}^{a}\left\{f_{i j}(t) q_{j}+\sum_{k \neq j} f_{i k}(t) q_{k j}\right\} \\
& \leqslant\left\{\sum_{i} g_{i}^{a} f_{i j}(t)\right\} g_{j}+\sum_{k \neq j}\left\{\sum_{i} g_{i}^{a} f_{i k}(t)\right\} q_{k j} \\
& \leqslant g_{j}^{a} q_{j}+\sum_{k \neq j} g_{k}^{a} q_{k j} \leqslant 2 g_{j}^{a} q_{j}
\end{aligned}
$$

$$
\mathrm{H}_{j}^{a}(t)=\sum_{i} g_{i}^{a}\left[\delta_{i j}-f_{i j}(t)\right]=-\sum_{i} g_{i}^{a} \int_{0}^{t} f_{i j}^{\prime}(s) d s=-\int_{0}^{t} \sum_{i} g_{i}^{a} f_{i j}^{\prime}(s) d s
$$

by (6.27) and bounded convergence. Upon differentiation we obtain (6.23). Starting with (6.23), substituting from (II) again,. and relying on (6.27) for the interchange of summations, we obtain

$$
\begin{aligned}
\eta_{j}^{a}(t)=-\sum_{i} g_{i}^{a}\left\{\sum_{k} f_{i k}(t) q_{k j}\right\}=-\sum_{k}\left\{\sum_{i} g_{i}^{a} f_{i k}(t)\right\} q_{k j} & =-\sum_{k}\left\{g_{k}^{a}-\mathrm{H}_{k}^{a}(t)\right\} q_{k j} \\
& =-\sum_{k} \int_{t}^{\infty} \eta_{k}^{a}(s) q_{k j} d s
\end{aligned}
$$

Letting $t \downarrow 0$ we obtain (6.24) by Corollary 1 to Theorem 6.3. Furthermore, the series in (6.25) converges absolutely, having only one negative term, for almost every $t$ and the summation and integration in the last member of (6.29) can be interchanged, proving the absolute continuity of $\eta_{j}^{a}$ together with (6.25).

THEOREM 6.8. $\xi_{j}^{a}$ is absolutely continuous in $\mathbf{T}$; we have

$$
\frac{d}{d t} \xi_{j}^{a}(t)+\xi_{j}^{a}(t) q_{j}=\sum_{i} \xi_{i}^{a}(s) v_{i j}(t-s)
$$

for almost every $t$ and every $s$ in $(0, t)$, where

$$
\begin{gathered}
v_{i j}(t)=p_{i j}^{\prime}(t)+p_{i j}(t) q_{j} ;\left({ }^{1}\right) \\
\frac{d}{d t} \xi_{j}^{a}(t) \geqslant \sum_{i} \xi_{i}^{a}(t) q_{i j}
\end{gathered}
$$

for almost every $t$. For each $a$ in $\mathbf{A}$ and $j$ in $\mathbf{I}^{a}$, the following three propositions are equivalent:

(i) $\left(I I_{i j}\right)$ holds for every $i$ in $\mathbf{I}^{\alpha}$;

(ii) Equality holds in (6.32) for almost every $t$;

(iii) $\xi_{j}^{a}(0)=\eta_{j}^{a}(0)=0$.

(1) See $\left[1 ; \S\right.$ II. 16] for a discussion of $v_{i j}$. 
Proof. Using [1; (II.16.2)] we write for each $t>0$ and $0<s<t$,

$$
\xi_{j}^{a}(t)=\sum_{i} \xi_{i}^{a}(s) p_{i j}(t-s)=\sum_{i} \xi_{i}^{a}(s)\left[\delta_{i j} e^{-q_{j}(t-s)}+\int_{0}^{t-s} v_{i j}(u) e^{-q_{j}(t-s-u)} d u\right]
$$

Since $\sum_{i} \xi_{i}^{a}(s)=1$ by $(4.20)$, this shows that $\xi_{j}^{a}$ is absolutely continuous in $\mathbf{T}^{0}$, hence in $\mathbf{T}$ by its continuity at zero. Multiplying (6.33) through by $e^{a_{j} t}$ and using Fubini's theorem on differentiation, we obtain (6.30) for almost every $t$. Substituting the inequality

$$
v_{i j}(t-s) \geqslant \sum_{k \neq j} p_{i k}(t-s) q_{k j}
$$

into (6.30) and using (4.21) we obtain (6.32).

If (i) is true, then equality holds in (6.34) by the definition of $v_{i j}$ and the preceding substitution leads to equality in (6.32). Thus (i) implies (ii). Conversely if (ii) is true, then for almost every $t$ and $0<s<t$,

$$
\frac{d}{d t} \xi_{j}^{a}(t)+\xi_{j}^{a}(t) q_{j}=\sum_{k \neq j} \xi_{k}^{a}(t) q_{k j}=\sum_{k \neq j}\left[\sum_{i} \xi_{i}^{a}(s) p_{i k}(t-s)\right] q_{k j}=\sum_{i} \xi_{i}^{a}(s) \sum_{k \neq j} p_{i k}(t-s) q_{k j} .
$$

Comparing (6.30) with (6.35), we see that equality must hold in (6.34) whenever $\xi_{i}^{a}(s)>0$. For each $i$ in $\mathbf{I}^{a}$ this is the case for every sufficiently large $s$. It follows that equality holds for every $i$ in $\mathbf{I}^{a}$ and every $t-s$, that is, $\left(\mathrm{II}_{i j}\right)$ holds for every $i$ in $\mathbf{I}^{a}$. Thus (ii) implies (i) and we have proved the equivalence of (i) and (ii).

It follows from (6.32) that for each $u>0$ :

$$
\xi_{j}^{a}(u)-\xi_{j}^{a}(0)-\sum_{i} \int_{0}^{u} \xi_{i}^{a}(t) q_{i j} d t=\int_{0}^{u}\left[\frac{d}{d t} \xi_{j}^{a}(t)-\sum_{i} \xi_{i}^{a}(t) q_{i j}\right] d t \geqslant 0 .
$$

Since $\lim _{u \rightarrow \infty} \xi_{j}^{a}(u)=0$ as a consequence of Theorem 6.1, we have upon letting $u \rightarrow \infty$ and using (6.24):

$$
-\xi_{j}^{a}(0)+\eta_{j}^{a}(0)=-\xi_{j}^{a}(0)-\sum_{i} g_{i}^{a} q_{i j} \geqslant 0
$$

If (iii) is true, then there is equality in (6.36) and hence also in (6.32): Thus (iii) implies (ii). Conversely if (ii) is true, the same argument shows that there is equality in (6.37). To prove that $\xi_{j}^{a}(0)=0$, let us suppose the contrary. There exist $i$ and $t$ such that $L_{i}^{a}(t)>0$, hence we have, as a consequence of the Corollary to Theorem 4.3 and Theorem 4.4:

$$
\mathbf{P}_{i}\left\{\tau^{a} \leqslant t ; x_{s}=j \text { for all } s \text { in }\left(\tau^{a}, t\right)\right\} \geqslant \int_{0}^{t} \xi_{j}^{a}(0) e^{-a_{j}(t-s)} d L_{i}^{a}(s)>0 .
$$

This means that there is positive probability that $x_{0}=i, x_{t}=j$ and that the last dis5-632932 Acta mathematica. 110. Imprimé le 15 octobre 1963. 
continuity of the sample function before time $t$ is a "pseudo-jump" from $\infty^{a}$ and not a jump. By [1; Theorem II.14.4] (1) this cannot happen under (i). Since (i) and (ii) have been shown to be equivalent, we conclude that (ii) implies (iii) and Theorem 6.8 is completely proved.

Remark. If (6.7) holds, then dividing through by $t$ and letting $t \downarrow 0$ we obtain $\eta_{j}^{a}(0) \geqslant \xi_{j}^{a}(0)$ in general, and $\eta_{j}^{a}(0)=\xi_{j}^{a}(0)$ if $\sigma^{a b}(0)<\infty$ for every $a$ and $b$.

\section{§ 7. The Dual Chain}

In this section we study the notion of a dual chain. Combining it and the results of $\S 6$ we shall derive a representation of $\left\{\xi_{j}\right\}$ when the method of $\S 5$ fails, and discuss the case left open there, namely the alternative (i) in Theorem 5.5. This is the case where the boundary behavior, even under the most stringent set of assumptions made here, is still not fully understood. It should be stressed that the dual chain studied here is more an analytical device than a genuinely probabilistic one. The latter would be that of a reversed chain as has been introduced in simpler cases (see [2]) and would involve an investigation of the sample function as the direction of time is reversed. This has not yet been done in a satisfactory manner and the results below serve only as a sort of vague reflection of the true state of matters.

For a few moments Assumptions A, B, and C (without B') will suffice. For each $\boldsymbol{a}$ in $\mathbf{A}$ we set

$$
\tilde{p}_{j i}^{a}(t)=\frac{g_{i}^{a} p_{i j}(t)}{g_{i}^{a}}
$$

The matrix $\left(\tilde{p}_{i j}^{a}\right),(j, i) \in \mathbf{I}^{a} \times \mathbf{I}^{a}$, will be called the $a$-dual to $\left(p_{i j}\right)$. Where this dual matrix is concerned the index set will be $\mathbf{I}^{a}$ without specific mention.

THEOREM 7.1. For each a in $\mathbf{A},\left(\tilde{p}_{j i}^{a}\right)$ isa strictly substochastic transition matrix. Its initial derivative matrix $\left(\tilde{q}_{j i}^{a}\right)$ and the corresponding minimal solution $\left(f_{j i}^{a}\right)$ are given as follows:

$$
\begin{gathered}
\tilde{q}_{j i}^{a}=\frac{g_{i}^{a} q_{i j}}{g_{j}^{a}} \\
\tilde{f}_{j i}^{a}(t)=\frac{g_{i}^{a} f_{i j}(t)}{g_{j}^{a}} .
\end{gathered}
$$

(1) I take this opportunity to acknowledge that the argument in the first few lines of p. 223 in [1] is inadequate for an instantaneous state $k$, as pointed out to me by S. Orey. This has been corrected both by him and by myself but the revision is too long to be included here. For the purpose here, where all states are stable, the proof given in [1] is correct. 
The matrix $\left(\tilde{q}_{j i}^{a}\right)$ is stochastic if and only if $\eta_{j}^{a}(0)=0$ for every $j$ in $\mathbf{I}^{a}$. We have

$$
\tilde{L}_{j}^{a}(t)=1-\sum_{i} \tilde{f}_{j i}^{a}(t)=\frac{\mathbf{H}_{j}^{a}(t)}{g_{j}^{a}}
$$

the function $\tilde{L}_{j}^{a}$ has a continuous derivative $\tilde{l}_{j}^{a}$ satisfying

$$
\sum_{i}\left[g_{i}^{a} \tilde{l}_{i}^{a}(t)\right] f_{i j}(s)=g_{j}^{a} \tilde{l}_{j}^{a}(t+s)
$$

Proof. It is easy to see that $\left(\tilde{p}_{j i}^{a}\right)$ satisfies the semi-group property corresponding to (2.2). Next we have by definition

$$
\sum_{i} \tilde{p}_{j i}^{a}(t)=\frac{1}{g_{j}^{a}} \sum_{i} \int_{0}^{\infty} \xi_{i}^{a}(s) p_{i j}(t) d s=\frac{1}{g_{j}^{a}} \int_{t}^{\infty} \xi_{j}^{a}(s) d s \leqslant 1 .
$$

In fact if $j \in \mathbf{I}^{a}$, then $\xi_{j}^{a}(\cdot)>0$ by the Appendix, so that there is strict inequality in (7.6) for every $t>0$. This and trivial inspection show that $\left(\tilde{p}_{j i}^{a}\right)$ is a strictly substochastic standard transition matrix and (7.2) follows at once from (7.1). It is easy to verify that $\left(f_{i j}^{a}\right)$ as defined by (7.3) is the minimal solution to the two systems of Kolmogorov differential equations (I) and (II) in $\S 2$ when $\left(q_{i j}\right)$ there is replaced by $\left(\tilde{q}_{i j}^{a}\right)$. Moreover we have

$$
\sum_{i} \tilde{q}_{j i}^{a}=\frac{1}{g_{j}^{a}} \sum_{i} g_{i}^{a} q_{i j}=-\frac{\eta_{j}^{a}(0)}{g_{j}^{a}} \leqslant 0
$$

by (6.24). The equation (7.4) follows at once from (7.3) and (6.2). Hence by Theorem 6.2 , we have

$$
\tilde{l}_{j}^{a}(t)=\frac{\eta_{j}^{a}(t)}{g_{j}^{a}}
$$

and (7.5) follows from (6.5). Theorem 7.1 is proved.

A homogeneous Markow chain $\tilde{x}^{a}=\left\{\tilde{x}^{a}(t), t \in \mathbf{T}\right\}$ having $\mathbf{I}^{a}$ as its state space and the stochastic completion of $\left(\tilde{p}_{j i}^{a}\right)$ as its transition matrix is called an a-dual to $x$. If $\eta_{j}^{a}(0)=0$ for every $j$ in $I^{a}$, then it satisfies an assumption corresponding to Assumption A for $x$ and so we may proceed to apply the preceding theory to it. However, to encompass as large a state space as possible we must take at suitable mixture of the indices $a$ as follows.

Let

$$
\tilde{I}=\bigcup_{a \in \mathbf{A}} \mathbf{I}^{a}
$$

$I$ is the state space of the post- $\tau$ process under Assumptions $\mathbf{A}$ and $\mathbf{B}$; it is clear that it is a $\prod$-stochastically closed subset of $\mathbf{I}$. Let 


$$
L^{a}=\sum_{i} \gamma_{i} L_{i}^{a},
$$

where $\gamma$ is the initial distribution of $x$, and set

$$
h_{j}=\sum_{a \in \mathbf{A}} L^{a}(\infty) g_{j}^{a} .
$$

We now introduce the following assumption which is essentially the dual of Assumption $\mathbf{A}$.

Assumption Ã. The second system of Kolmogorov differential equations holds.

Theorem 7.2. (Under Assumptions A, B, C and Ã.) We have $h_{j}<\infty$ for every $j$ in $\mathbf{I}$, and $h_{j}>0$ if and only if $j \in \tilde{\mathbf{I}}$. Furthermore,

$$
\begin{aligned}
& \sum_{i} h_{i} p_{i j}(t) \leqslant h_{j}, \\
& \sum_{i} h_{i} q_{i j}=0 .
\end{aligned}
$$

Proof. The first assertion follows from (5.1) upon integration over T:

$$
h_{j}=\int_{0}^{\infty} \sum_{i} \gamma_{i}\left[p_{i j}(t)-f_{i j}(t)\right] d t<\infty,
$$

since $\int_{0}^{\infty} \sum_{i} \gamma_{i} p_{i j}(t) d t<\infty$ for a nonrecurrent state $j$. By the definition of $\mathbf{A}$, for each $a$ in $\mathbf{A}$ there exists an $i$ such that $\gamma_{i}>0$ and $L_{i}^{a}(\infty)>0$, hence $L^{a}(\infty)>0$. By Theorem 6.1, $g_{j}^{a}>0$ if and only if $j \in \mathbf{I}^{a}$. These remarks prove that $h_{j}>0$ if and only if $j \in \tilde{\mathbf{I}}$. Next, we have

$$
\sum_{i} h_{i} p_{i j}(t)=\sum_{a} L^{a}(\infty) \sum_{i} g_{i}^{a} p_{i j}(t) \leqslant \sum_{a} L^{a}(\infty) g_{j}^{a}=h_{j} .
$$

Finally, we have by (6.24) and Theorem 6.8, under Assumption Ã:

$$
\sum_{i} h_{i} q_{i j}=\sum_{a} L^{a}(\infty) \sum_{i} g_{i}^{a} q_{i j}=0,
$$

the interchange of the repeated summation being justified since $\sum_{i}\left|h_{i} q_{i j}\right| \leqslant 2 h_{j} q_{j}<\infty$ by (6.26). Theorem 7.2 is proved.

$$
\begin{gathered}
\tilde{p}_{j i}(t)=\frac{h_{i} p_{i j}(t)}{h_{j}}, \\
\tilde{q}_{j i}=\frac{h_{i} q_{i j}}{h_{j}}, \quad-\tilde{q}_{j j}=\tilde{q}_{j}=q_{j},
\end{gathered}
$$




$$
\begin{gathered}
\tilde{r}_{j i}=\frac{\left(1-\delta_{j i}\right) \tilde{q}_{j i}}{\tilde{q}_{j}}=\frac{\left(1-\delta_{i j}\right) h_{i} q_{i j}}{h_{j} q_{j}} \\
\tilde{f}_{j i}(t)=\frac{h_{i} f_{i j}(t)}{h_{j}}
\end{gathered}
$$

The matrix $\tilde{\Pi}=\left(\tilde{p}_{j i}\right),(j, i) \in \tilde{\mathbf{I}} \times \tilde{\mathbf{I}}$, will be called the dual transition matrix to $\left(p_{i j}\right)$; similarly for $\tilde{Q}, \tilde{\mathrm{P}}$ and $\tilde{\Phi}$. A homogeneous Markov chain $\tilde{x}=\{\tilde{x}(t), t \in \mathbf{T}\}$ having $\tilde{\mathbf{I}}$ as its state space and $\tilde{\Pi}_{\tilde{\theta}}$ as its transition matrix will be called the dual chain to $x$. By virtue of (7.11), the matrices $\tilde{Q}$ and $\tilde{P}$ are stochastic and so the dual chain satisfies the assumption corresponding to Assumption $A$ and we can define its jump chain $\tilde{\chi}=\left\{\tilde{\chi}_{n}\right\}$, its Martin boundary $\tilde{\mathbf{B}}$ and the passable part $\tilde{\mathbf{B}}$. The assumption corresponding to Assumption B, which we now make, is as follows.

Assumptons $\tilde{\mathrm{B}}, \tilde{\mathrm{B}}$. The passable part of the dual boundary is completely atomic.

These atoms will be denoted by $\{\tilde{\infty} \tilde{a}, \tilde{a} \in \tilde{\mathbf{A}}\}$. Assumption $\tilde{\mathbf{B}}$ becomes Assumption $\tilde{\mathbf{B}}^{\prime}$ iff $\tilde{\mathbf{A}}$ is a finite set. Under Assumption $\tilde{\mathbf{B}}^{\prime}$ we may and shall replace the definition (7.9) by the simpler one:

$$
h_{j}=\sum_{a} g_{j}^{a}
$$

It is clear that Theorem 7.2 remains valid after this replacement.

Theorem 7.3. Under Assumptions $\tilde{\mathrm{A}}$ and $\tilde{\mathrm{B}}$ :

$$
p_{i j}(t)=f_{i j}(t)+\sum_{\tilde{a} \in \tilde{\mathbf{A}}} \int_{0}^{t} k_{i}^{\tilde{a}}(s) \psi_{j}^{\tilde{a}}(t-s) d s
$$

where

$$
\begin{aligned}
& \sum_{j} p_{i j}(s) k_{j}^{\tilde{a}}(t)=k_{i}^{\tilde{a}}(s+t) \\
& \sum_{i} \psi_{i}^{\tilde{a}}(s) f_{i j}(t)=\psi_{j}^{\tilde{a}}(s+t)
\end{aligned}
$$

Proof. Theorem 5.1 applied to the dual transition matrix yields:

$$
\tilde{p}_{j i}(t)=\tilde{f}_{j i}(t)+\sum_{\tilde{a} \in \tilde{\mathbf{A}}} \int_{0}^{t} \tilde{l}_{j}^{\tilde{a}}(s) \tilde{\xi}_{i}^{\tilde{a}}(t-s) d s,
$$

where

$$
\begin{gathered}
\sum_{j} \tilde{f}_{i j}(t) \tilde{l}_{j}^{\tilde{a}}(s)=\tilde{l}_{i}^{\tilde{a}}(t+s), \\
\sum_{i} \tilde{\xi}_{i}^{\tilde{a}}(t) \tilde{p}_{i j}(s)=\tilde{\xi}_{j}^{\tilde{a}}(t+s),
\end{gathered}
$$


these formulas being the duals of (4.4) and (4.21) respectively. Putting

$$
\begin{gathered}
h_{j} \tilde{l}_{j}^{\tilde{a}}(\cdot)=\psi_{j}^{\tilde{a}}(\cdot), \\
h_{i}^{-1} \tilde{\xi}_{i}^{\tilde{a}}(\cdot)=k_{i}^{\tilde{a}}(\cdot),
\end{gathered}
$$

and substituting from (7.12) and (7.15) we obtain (7.17) and (7.18). Theorem 7.3 is proved.

Clearly the coexistence of the two formulas (5.1) and (7.16) has interesting implications. However, due to evident technical difficulties more stringent assumptions than those needed for both formulas will be invoked in the next theorem. We must also introduce a new definition.

A passable atomic boundary point $\infty^{a}$ is called nonrepeatable iff for every $i \in \tilde{\mathbf{I}}$ we have $L_{i}^{a}(\infty)=0$; otherwise it is called repeatable. Let the subset of $\mathbf{A}$ corresponding to nonrepeatable boundary points be $\mathbf{A}_{0}$. Such a boundary point $\infty^{a}$ is reached exactly once on $\Delta^{a}$, and is never reached again after the first infinity. It is inessential according to the definition in $\S 5$, indeed $L^{b a} \equiv 0$ for every $b$ in $\mathbf{A}$. It is trivial to construct a nonrepeatable boundary point: we need only start the Markov chain with an ascending escalator and hitch on an open Markov chain, say a descending escalator, with a disjoint state space.

THEOREM 7.4. Under Assumptions A, B', $\tilde{\mathrm{A}}, \tilde{\mathrm{B}}^{\prime}$ and $\mathrm{C}$, there exist nondecreasing, bounded functions $\boldsymbol{M}^{a \tilde{a}},(a, \tilde{a}) \in \mathbf{A} \times \tilde{\mathbf{A}}$, such that for every $a$ in $\mathbf{A}, j$ in $\mathbf{I}$ and $\boldsymbol{t}$ in $\mathbf{T}$ we have

$$
\xi_{j}^{a}(t)=\sum_{\tilde{a} \in \tilde{\mathbf{A}}} \int_{0}^{t} \psi_{j}^{\tilde{a}}(t-s) d M^{a \tilde{a}}(s) .
$$

Proof. Comparing (5.1) and (7.16) we have

$$
\sum_{b \in \mathbf{A}} \int_{0}^{t} l_{i}^{b}(s) \xi_{j}^{b}(t-s) d s=\sum_{\tilde{b} \in \tilde{\mathbf{A}}} \int_{0}^{t} k_{i}^{\tilde{b}}(s) \psi_{j}^{\tilde{b}}(t-s) d s,
$$

where both $\mathbf{A}$ and $\tilde{\mathbf{A}}$ are finite sets. According to the Corollary to Theorem 4.1, for each $a$ there exists a sequence $\left\{i_{n}\right\}$ such that for every $b$ in $\mathbf{A}$,

$$
\lim _{n \rightarrow \infty} \int_{0}^{t} l_{i_{n}}^{b}(s) \xi_{j}^{b}(t-s) d s=\delta^{b a} \xi_{j}^{a}(t)
$$

If $a \notin \mathbf{A}_{0}$, namely if $\infty^{a}$ is repeatable, then we may choose $\left\{i_{n}\right\}$ so that $i_{n} \in \bar{I}$ for every $n$ and (7.25) holds with $i=i_{n}$. Now if we integrate this equation over $\mathbf{T}$ and take only the term corresponding to the index $\tilde{a}$ on the right side, we have by (7.22) and (7.23): 


$$
\int_{0}^{\infty} k_{i}^{\tilde{a}}(s) d s \int_{0}^{\infty} \psi_{j}^{\tilde{a}}(s) d s=\int_{0}^{\infty} k_{i}^{\tilde{a}}(s) d s h_{j} \tilde{L}_{j}^{\tilde{a}}(\infty) \leqslant \sum_{b \in \mathbf{A}} L_{i}^{b}(\infty) g_{j}^{b} \leqslant h_{j}
$$

Choosing $j$ such that $\tilde{L}_{j}^{\tilde{a}}(\infty)>0$, and putting $K_{i}^{\tilde{a}}(t)=\int_{0}^{t} k_{i}^{\tilde{a}}(s) d s$, we see that

$$
K_{i}^{\tilde{a}}(\infty) \leqslant \frac{1}{L_{j}^{\tilde{a}}(\infty)}<\infty
$$

Thus the family of nondecreasing functions $\left\{K_{i}^{\tilde{a}}, i \in \mathrm{I}\right\}$ has a uniformly bounded total variation and so is weakly compact. It follows that there is a subsequence $\left\{i_{n}^{\prime}\right\}$ (depending on $\tilde{a}$ ) of $\left\{i_{n}\right\}$ (depending on $a$ ) for which $K_{i^{\prime} n}^{\tilde{a}}(\cdot)$ converges weakly to a limit $M^{\tilde{a}}(\cdot)$ which is nondecreasing and bounded with $M^{\tilde{a}}(0)=0$. Applying this result to (7.25), noting the continuity of $\psi_{j}^{\tilde{a}}$ and using (7.26), we obtain (7.24) for every $a \notin \mathbf{A}_{\mathbf{0}}$.

It remains to prove (7.24) for $a \in \mathbf{A}_{0}$. Since $L^{a b}(\infty)=0$ for $a \in \mathbf{A}$ and $b \in \mathbf{A}_{0}$, we may rewrite $(5.20)$ as follows, omitting the index $j$ :

$$
\xi^{a}(t)=\zeta^{a}(t)+\sum_{b \in \mathbf{A} \backslash \mathbf{A}_{0}} \int_{0}^{t} \xi^{b}(t-s) d L^{a b}(s) .
$$

Substituting from the proved part of (7.24), we have

$$
\xi^{a}(t)=\zeta^{a}(t)+\sum_{\tilde{a} \in \tilde{\mathbf{A}}} \int_{0}^{t} \psi^{\tilde{a}}(t-s) d N^{\tilde{a} \tilde{a}}(s)
$$

where

$$
N^{a \tilde{a}}=\sum_{b \in \mathbf{A} \backslash \mathbf{A}_{0}}\left(L^{a b} * M^{b \tilde{a}}\right)
$$

and * denotes a convolution. Next, the equation (5.12) becomes, after substituting from (7.15) and noting that $\zeta_{i}^{a}(\cdot) \equiv 0$ if $i \notin \tilde{\mathbf{I}}$,

$$
\sum_{i \in \tilde{\mathbf{I}}} \tilde{f}_{j i}(t)\left[h_{i}^{-1} \zeta_{i}^{a}(s)\right]=h_{j}^{-1} \zeta_{j}^{a}(t+s) \quad(j \in \tilde{\mathbf{I}})
$$

Using the definition given in $\S 4$, the set $\left\{h_{i}^{-1} \zeta_{i}^{a}(\cdot)\right\}$ is an exit solution for $\tilde{\Phi}$ for each $a$, since $\zeta_{i}^{a}(0)=0$ by (7.29) and Theorem 6.8; moreover by (5.20),

$$
\int_{0}^{\infty} h_{i}^{-1} \zeta_{i}^{a}(t) d t \leqslant h_{i}^{-1} \int_{0}^{\infty} \xi_{i}^{a}(t) d t \leqslant 1
$$

Hence according to Theorem 4.2 applied to the dual chain, and using (7.22):

$$
\zeta_{i}^{a}(t)=\sum_{\tilde{a} \in \tilde{\mathbf{A}}} c^{a \tilde{a}} \psi_{i}^{\tilde{a}}(t)
$$


where $0 \leqslant c^{a \tilde{a}} \leqslant 1$. Substituting into $(7.30)$, we see that (7.24) holds with

Theorem 7.4 is proved.

$$
M^{a \bar{a}}=N^{a \bar{a}}+c^{a \bar{a}} \varepsilon
$$

A set of nonnegative functions $\left\{u_{i}(\cdot)\right\}$ with $u_{i}(0)=0$ for every $i$ and satisfying the system of functional equations

$$
\sum_{i} u_{i}(s) f_{i j}(t)=u_{j}(s+t)
$$

will be called an entrance solution for $\Phi$. Under Assumption $\tilde{A}$, the sets $\left\{\zeta_{i}^{a}(\cdot)\right\}$ and $\left\{\eta_{i}^{a}(\cdot)\right\}$ defined in Theorems 5.2 and 6.2 are entrance solutions for $\Phi$. We have seen in the above how an entrance solution for $\Phi$ corresponds to an exit solution for $\tilde{\Phi}$. The set $\left\{\psi^{\tilde{a}}, \tilde{a} \in \tilde{\mathbf{A}}\right\}$ forms an extreme base for the space of entrance solutions restricted to $\tilde{\mathbf{I}}$. In particular, we can express $\zeta$ and $\eta$ in terms of $\psi$.

COROLLARY TO THEOREM 7.4. We have for every $a$ and $j \in \mathbf{I}$;

$$
\begin{aligned}
\zeta_{j}^{a}(t) & =\sum_{\tilde{a}} M^{a \tilde{a}}(0+) \psi_{j}^{\tilde{a}}(t), \\
\eta_{j}^{a}(t) & =\sum_{\tilde{a}} M^{a \tilde{a}}(\infty) \psi_{j}^{\tilde{a}}(t) .
\end{aligned}
$$

Proof. (7.34) follows from the following calculation:

$$
\begin{aligned}
\zeta_{j}^{a}(t) & =\lim _{s \downarrow 0} \sum_{i} \sum_{\tilde{a}} \int_{0}^{s} \psi_{i}^{\tilde{a}}(s-u) f_{i j}(t-s) d M^{a \tilde{a}}(u) \\
& =\sum_{\tilde{a}} \lim _{s \downarrow 0} \int_{0}^{s} \psi_{j}^{\tilde{a}}(t-u) d M^{\tilde{a} \tilde{a}}(u)=\sum_{\tilde{a}} M^{a \tilde{a}}(0+) \psi_{j}^{a}(t) .
\end{aligned}
$$

To prove (7.35), we first integrate $(7.24)$ to obtain

$$
g_{j}^{a}=\sum_{\tilde{a}} M^{a \tilde{a}}(\infty) \int_{0}^{\infty} \psi_{j}^{\tilde{a}}(s) d s .
$$

Consequently

$$
\sum_{i} g_{i}^{a} f_{i j}(t)=\sum_{\tilde{a}} M^{\tilde{a}}(\infty) \int_{0}^{\infty} \psi_{j}^{\tilde{a}}(s+t) d s,
$$

and

$$
\mathrm{H}_{j}^{a}(t)=\sum_{\tilde{a}} M^{\tilde{a}}(\infty) \int_{0}^{t} \psi_{j}^{\bar{a}}(s) d s,
$$

from which (7.35) follows upon differentiation. 
On the basis of Theorem 7.4, we can express the probabilities in (5.5) and (5.6) in a suggestive way as follows:

$$
\begin{aligned}
\mathbf{P}^{a}\left\{\delta_{t}^{a} \leqslant s\right\} & =\sum_{\tilde{a}} \int_{0}^{s} \psi_{*}^{\tilde{a}}(t-u) d M^{\tilde{a}}(u) \\
\mathbf{P}^{a}\left\{\delta_{t}^{a} \leqslant s ; x_{t}^{a}=j\right\} & =\sum_{\tilde{a}} \int_{0}^{s} \psi_{j}^{\tilde{a}}(t-u) d M^{a \tilde{a}}(u) \\
& =\sum_{\tilde{a}} \int_{0}^{s} \frac{\psi_{j}^{\tilde{a}}(t-u)}{\psi_{*}^{\tilde{a}}(t-u)} \psi_{*}^{\tilde{a}}(t-u) d M^{a \tilde{a}}(u) .
\end{aligned}
$$

Thus the last non-jump discontinuity before time $t$ in the post- $\tau^{\alpha}$ process enjoys properties similar to that of the last exit time from an ordinary state before time $t$, discussed in [2]. Starting from this it is possible to discuss the reversed chain rigorously as a probabilistic object. but we shall not pursue the matter further here.

We can also use Theorem 7.4 to obtain criteria for either alternative in Theorem 5.5. Write $\psi_{*}^{\tilde{a}}(\cdot)=\sum_{j} \psi_{j}^{\tilde{a}}(\cdot)$ as in $(6.15)$.

THEOREM 7.5. Under Assumptions A and $\mathbf{B}^{\prime}$, if $\eta_{*}^{a}(0)<\infty$ (or equivalently $\left.\sigma^{a}(0)<\infty\right)$ then $\varrho^{a}(0)=0$. Case (ii) of Theorem 5.5 obtains if in each essential class of indices, there exists at least one index a for which $\eta_{*}^{a}(0)<\infty$. Under the additional Assumptions $\tilde{\mathrm{A}}$ and $\tilde{\mathrm{B}}$ this is the case if $\psi_{*}^{\tilde{a}}(0)<\infty$ for every $\tilde{a}$. On the other hand, if $\psi_{*}^{\tilde{a}}(0)=\infty$ for every $\tilde{a}$, then case (i) of Theorem 5.5 obtains.

Proof. It follows from (5.10) that

$$
\varrho^{a}(t)=\lim _{u \downarrow 0} u^{-1} \int_{0}^{u} \sum_{i} \xi_{i}^{a}(s) L_{i}(t-s) d s .
$$

By (6.7), we have $\int_{0}^{u} \xi_{i}^{a}(s) d s \leqslant \int_{0}^{u} \eta_{i}^{\dot{a}}(s) d s$, hence by $(6.19)$ :

$$
\varrho^{a}(t) \leqslant \lim _{u \downarrow 0} u^{-1} \int_{0}^{u}\left[\eta_{*}^{a}(s)-\eta_{*}^{a}(t)\right] d s=\eta_{*}^{a}(0)-\eta_{*}^{a}(t)
$$

since $\eta_{*}^{a}(s)$ is nondecreasing as $s \downarrow 0$. Hence if $\eta_{*}^{a}(0)<\infty$, then

$$
\varrho^{a}(0)=\lim _{t \downarrow 0} \varrho^{a}(t) \leqslant \lim _{t \downarrow 0}\left[\eta_{*}^{a}(0)-\eta_{*}^{a}(t)\right]=0 .
$$

Since $\mathbf{A}$ is finite, (5.21) holds and

$$
\sum_{b} L^{a b}(0+)=0
$$


If $a$ is essential this excludes the possibility of $(5.26)$ for the essential class to which $a$ belongs. If this is so for each such class, Theorem 5.5 asserts that case (ii) there occurs. Finally, if $\psi_{*}^{\tilde{a}}(0)<\infty$ for every $\tilde{a}$, then $\eta_{*}^{a}(0)<\infty$ by (7.35) and we have case (ii) by the above.

Now suppose the other extreme: $\psi_{*}^{\tilde{a}}(0)=\infty$ for every $\tilde{a}$. As before we have

$$
\sum_{i} \psi_{i}^{\tilde{a}}(s) L_{i}(t-s)=\psi_{*}^{\bar{a}}(s)-\psi_{*}^{\bar{a}}(t),
$$

and consequently by (7.24):

$$
\sum_{i} \xi_{i}^{a}(s) L_{i}(t-s)=\sum_{\tilde{a}} \int_{0}^{s}\left[\psi_{*}^{\tilde{a}}(s-u)-\psi_{*}^{\tilde{a}}(t-u)\right] d M^{a \tilde{a}}(u)
$$

It follows that

$$
\sum_{\tilde{a}} M^{\alpha \tilde{a}}(0+)\left[\psi_{*}^{\tilde{a}}(s)-\psi_{*}^{\tilde{a}}(t)\right] \leqslant 1 .
$$

As $s \downarrow 0$ this implies $M^{\tilde{a}}(0+)=0$ for every $\tilde{a}$ and so by (7.34), $\zeta_{j}^{a}(t)=0$ for every $j$ and $t$. Hence by (5.11), $\varrho^{a}(t)=1$ for every $t$ and so $\varrho^{a}(0)=1$. This means $\sum_{t} L^{a b}(0+)=1$ by (5.21) and we have case (i) of Theorem 5.3.

\section{§ 8. The Construction Theorem}

There is a basic connection between Theorems 6.3 and 7.4 which leads to a solution of the construction problem. In this section we make full use of the method of Laplace transforms.

Taking Laplace transforms in (6.7) and using matrix notation, we have

$$
\hat{\eta}(\lambda)=[I+\lambda \hat{\Sigma}(\lambda)] \hat{\xi}(\lambda),
$$

where $\hat{\Sigma}(\lambda)$ is the matrix $\left(\hat{\sigma}^{a b}(\lambda)\right),(a, b) \in \mathbf{A} \times \mathbf{A}$. We are under Assumptions $\mathbf{B}^{\prime}$ and $\tilde{\mathbf{B}}^{\prime}$ so that both $\mathbf{A}$ and $\tilde{\mathbf{A}}$ are finite sets. We have by (7.35),

$$
\hat{\eta}(\lambda)=\boldsymbol{M} \hat{\psi}(\lambda)
$$

where $M=\left(M^{a \tilde{a}}(\infty)\right),(a, \tilde{a}) \in \mathbf{A} \times \tilde{\mathbf{A}}$, is a constant matrix. For a few moments let $\theta_{0}^{\tilde{a} b}$ and $\sigma_{0}^{\tilde{a} b}$ denote the quantities $\theta^{a b}$ and $\sigma^{a b}$ in (6.10) and (6.11) when $\eta^{a}$ is replaced by $\psi^{\tilde{a}}$. Since both $\left\{\eta_{i}^{a}(\cdot)\right\}$ and $\left\{\psi_{i}^{\tilde{a}}(\cdot)\right\}$ are entrance solutions, the properties of $\theta^{a b}$ and $\sigma^{a b}$ deriving from the fact that $\eta^{a}$ is an entrance solution hold also for $\theta_{0}^{\tilde{a} b}$ and $\sigma_{0}^{\tilde{a} b}$. Finally, let

$$
u^{\tilde{a} a}(\lambda)=\lambda \hat{\sigma}_{0}^{\bar{a} a}(\lambda)
$$

and $U(\lambda)$ be the matrix $\left(u^{\tilde{a} a}(\lambda)\right),(\tilde{a}, a) \in \tilde{\mathbf{A}} \times \mathbf{A}$. 
Lемма. For each $\tilde{a}$ and $a, \frac{d}{d \lambda} u^{\tilde{a} a}(\lambda)$ is a completely monotonic function of $\lambda$.

Proof. We have by (6.12) and a simple calculation:

$$
\begin{aligned}
\frac{d}{d \lambda} u^{\tilde{a} a}(\lambda) & =\int_{0}^{\infty} e^{-\lambda t}(1-\lambda t) \sigma_{0}^{\tilde{a} a}(t) d t \\
& =\int_{0}^{\infty} e^{-\lambda t}(1-\lambda t) d t \int_{t}^{\infty} \theta_{0}^{\tilde{a} a}(s) d s=\int_{0}^{\infty} e^{-\lambda s} s \theta_{0}^{\tilde{a} a}(s) d s
\end{aligned}
$$

Since we have

$$
\theta_{0}^{\tilde{a} a}(s)=\frac{1}{s} \int_{0}^{s} \sum_{i} \psi_{i}^{\tilde{a}}(u) l_{i}^{a}(s-u) d u
$$

by Theorem 6.4 applied to $\psi$, the last member of $(8.4)$ is equal to $\sum_{i} \hat{\psi}_{i}^{\tilde{a}}(\lambda) \hat{l}_{i}^{a}(\lambda)$. Since $\hat{l}_{i}^{a}(\lambda) \leqslant 1$, and $\sum_{i} \psi_{i}^{\tilde{a}}(\lambda)<\infty$ by Theorem 6.5 applied to $\psi, \sum_{i} \hat{\psi}_{i}^{\tilde{a}}(\lambda) \hat{l}_{i}^{a}(\lambda)$ converges and is completely monotonic in $\lambda$ since each term is. The lemma is proved.

In terms of $\psi$, the equation (8.1) can be written as

$$
M \hat{\psi}(\lambda)=[I+M U(\lambda)] \hat{\xi}(\lambda)
$$

It is our object to study the solvability of (8.5) for $\hat{\xi}(\lambda)$. The folloving theorem is a general result about completely monotonic functions. Let us call a matrix of functions completely monotonic iff each element of the matrix is so.

THEOREM 8.1. Let $M$ be an $\mathbf{A} \times \tilde{\mathbf{A}}$ matrix with elements which are nonnegative constants, $M(\lambda)$ likewise with elements which are nonnegative functions of $\lambda ; U(\lambda)$ an $\tilde{\mathbf{A}} \times \mathbf{A}$ matrix with elements whose derivatives are completely monotonic functions of $\lambda$. Suppose that for each $\lambda$ we have

$$
M=[I+M U(\lambda)] M(\lambda),
$$

where $I$ is the $\mathbf{A} \times \mathbf{A}$ identity matrix. Then both $I+M U(\lambda)$ and $I+U(\lambda) M$ are invertible; we have

$$
M=M(\lambda)[I+U(\lambda) M]
$$

and the matrix $M(\lambda)$ is completely monotonic.

Proof. To show that $I+M U(\lambda)$ is invertible, suppose there exists a vector $v$ such that

$$
v[I+M U(\lambda)]=0
$$


Then by (8.6), $v M=0$ and consequently by (8.8), $v=0$. To show that $I+U(\lambda) M$ is invertible, suppose there exists a vector $w$ such that

$$
[I+U(\lambda) M] w=0
$$

Then

$$
[I+M U(\lambda)] M w=M[I+U(\lambda) M] w=0
$$

Since $I+M U(\lambda)$ is invertible as just shown, we have $M w=0$ and consequently $w=0$ by $(8.9) .(1)$

$$
\text { Since } \quad[I+M U(\lambda)] M=M[I+U(\lambda) M],
$$

it follows that $\quad M[I+U(\lambda) M]^{-1}=[I+M U(\lambda)]^{-1} M=M(\lambda)$

by (8.6), and so (8.7) is true. Finally, for $\delta>0$ consider

$$
\begin{aligned}
{[I+} & M U(\lambda+\delta)]\{M(\lambda+\delta)-M(\lambda)\}[I+U(\lambda) M] \\
& =[I+M U(\lambda+\delta)]\left\{[I+M U(\lambda+\delta)]^{-1} M-M[I+U(\lambda) M]^{-1}\right\}[I+U(\lambda) M] \\
& =M[I+U(\lambda) M]-[I+M U(\lambda+\delta)] M=M[U(\lambda)-U(\lambda+\delta)] M .
\end{aligned}
$$

Dividing through by $\delta$ and letting $\delta \downarrow 0$, we obtain

$$
[I+M U(\lambda)] M^{\prime}(\lambda)[I+U(\lambda) M]=-M U^{\prime}(\lambda) M
$$

Equivalently, by (8.6) and (8.7), we have

$$
-M^{\prime}(\lambda)=M(\lambda) U^{\prime}(\lambda) M(\lambda)
$$

For the sake of induction let us now suppose that

$$
(-1)^{m} M^{(m)}(\lambda) \geqslant 0 \quad(0 \leqslant m \leqslant n) .
$$

This is true for $m=0$ by hypothesis. Differentiating (8.10) $n$ times by Leibniz's rule, we have

$$
\begin{aligned}
(-1)^{n+1} M^{(n+1)}(\lambda)= & \sum_{0 \leqslant j+k \leqslant n} \frac{n !}{j ! k !(n-j-k) !} M^{(j)}(\lambda) U^{(n+1-j-k)}(\lambda) M^{(k)}(\lambda) \\
= & \sum_{0 \leqslant j+k \leqslant n} \frac{n !}{j ! k !(n-j-k) !}(-1)^{j} M^{(j)}(\lambda)(-1)^{n-j-k} \\
& \times U^{(n+1-j-k)}(-1)^{k} M^{k}(\lambda) \geqslant 0,
\end{aligned}
$$

(1) I am indebted to N. G. de Bruijn for the preceding proof. 
by the induction hypothesis and the hypothesis about $U(\lambda)$. Therefore $(8.11)$ is true also for $m=n+1$ and the induction is complete, proving that $M(\lambda)$ is completely monotonic.

THEOREM 8.2. There exists an $\mathbf{A} \times \tilde{\mathbf{A}}$ matrix $M(\lambda)$ such that

$$
\hat{\xi}(\lambda)=M(\lambda) \hat{\psi}(\lambda)\left({ }^{1}\right) \quad(0<\lambda<\infty),
$$

if and only if there exists a constant matrix $M$ such that

$$
\hat{\eta}(\lambda)=M \hat{\psi}(\lambda) \quad(0<\lambda<\infty)
$$

and such that $I+M U(\lambda)$ is invertible. In this case $M(\lambda)$ is completely monotonic and $M=M(0)$.

Proof. Suppose (8.12) holds, namely

$$
\hat{\xi}_{i}^{a}(\lambda)=\sum_{\tilde{a}} m^{a \tilde{a}}(\lambda) \hat{\psi}_{i}^{\tilde{a}}(\lambda)
$$

By (7.22) and the Corollary to Theorem 4.1, for each $\tilde{a} \in \tilde{\mathbf{A}}$ there exists a sequence $\left\{i_{n}\right\}$ in $\tilde{\mathbf{I}}$ such that

$$
\lim _{n \rightarrow \infty} h_{i_{n}}^{-1} \hat{\psi}_{i_{n}}^{\tilde{b}}(\lambda)=\delta^{\tilde{a} \tilde{b}} \quad(\tilde{b} \in \tilde{\mathbf{A}})
$$

It follows from this and (8.14) that

$$
m^{\alpha \tilde{a}}(\lambda)=\lim _{n \rightarrow \infty} h_{i_{n}}^{-1} \hat{\xi}_{i_{n}}^{a}(\lambda) \geqslant 0
$$

so that the matrix $M(\lambda)$ is automatically nonnegative for every $\lambda$. Next, there exists a constant matrix $M$ and a sequence $\left\{\lambda_{n}\right\}$ converging to zero such that $\lim _{n \rightarrow \infty} M\left(\lambda_{n}\right)=M$; each element $m^{a \tilde{a}}$ of $M$ is finite since by (8.14):

$$
m^{a \tilde{a}} \leqslant \frac{\hat{\xi}_{i}^{a}(0)}{\hat{\psi}_{i}^{\tilde{a}}(0)}=\frac{g_{i}^{a}}{h_{i} \tilde{L}_{i}^{\tilde{a}}(\infty)}<\infty .
$$

It follows that

$$
g_{i}^{a}=\sum_{\tilde{a}} m^{a \tilde{a}} \int_{0}^{\infty} \psi_{i}^{\tilde{a}}(s) d s ;
$$

and consequently as in the proof of (7.35) that

$$
\eta_{i}^{a}(t)=\sum_{\tilde{a}} m^{a \tilde{a}} \psi_{i}^{\tilde{a}}(t)
$$

(1) The $M(\cdot)$ here is the Laplace transform of the $M(\cdot)$ in Theorem 7.4. We have omitted the cumbersome $\wedge$ where confusion is unlikely. 
Taking Laplace transforms we obtain (8.13). Furthermore, substituting from (8.12) into (8.5), we have

$$
M \hat{\psi}(\lambda)=[I+M U(\lambda)] M(\lambda) \hat{\psi}(\lambda)
$$

Since the set $\left\{\psi^{\tilde{a}}(\lambda), \tilde{a} \in \tilde{\mathbf{A}}\right\}$ is linearly independent for each $\lambda$, a fact which is obvious from (8.15), it follows that

$$
M=[I+M U(\lambda)] M(\lambda) .
$$

Theorem 8.1 is therefore applicable to yield the conclusions that $I+M U(\lambda)$ is invertible and that $M(\lambda)$ is completely monotonic.

Conversely, suppose that (8.13) holds; then $M \geqslant 0$ by (8.15). If $I+M U(\lambda)$ is invertible, then

$$
\hat{\xi}(\lambda)=[I+M U(\lambda)]^{-1} M \hat{\psi}(\lambda),
$$

and so if we set

$$
M(\lambda)=[I+M U(\lambda)]^{-1} M
$$

we obtain (8.12) and $M(\lambda)$ is completely monotonic as before. Theorem 8.2 is proved.

Corollary 1. $\lim _{\lambda \rightarrow 0} M(\lambda)$ exists.

Proof. This follows from the uniqueness of the representation in (8.16).

We have formulated Theorem 8.2 in such a way as to stress the logical equivalence of two analytical propositions. Actually we know (8.12) is true under our assumptions by Theorem 7.4, hence the new fact that emerges is as follows.

COROLLARY 2. The matrix $I+\lambda \Sigma(\lambda)$ in (8.1), or equivalently the matrix $I+M U(\lambda)$ in (8.5), is invertible.

Let us recount the main steps of analysis up to this point. We are given a substochastic transition matrix $\Pi$ on the index set $\mathbf{I} \times \mathbf{I}$ to begin with. The initial derivative matrix $Q$ and the minimal solution $\Phi$ are then defined. Assumptions $A, \tilde{A}$, $\mathrm{B}^{\prime}$ and $\mathrm{C}$ are made. We then define $l, \xi$ and $\tilde{Q}$. Now Assumption $\tilde{\mathrm{B}}^{\prime}$ is made, and $\psi$ is defined. The following decomposition (or representation) formula ensues by virtue of Theorems 5.1 and 7.4:

$$
\hat{\Pi}(\lambda)=\hat{\Phi}(\lambda)+\hat{l}(\lambda) \hat{M}(\lambda) \hat{\psi}(\lambda)
$$

where $\hat{\Pi}(\lambda)=\left(\hat{p}_{i j}(\lambda)\right), \hat{\Phi}(\lambda)=\left(f_{i j}(\lambda)\right),(i, j) \in \mathbf{I} \times \mathbf{I}$, and where $\hat{\boldsymbol{M}}(\lambda)$ is written for the $\boldsymbol{M}(\lambda)$ in (8.12) in conformity with the rest of our notation. $U(\lambda)$ is defined through $l$ and $\psi$; finally let us write $\langle u, v\rangle=\sum_{i} u_{i} v_{i}$ if $u=\left\{u_{i}\right\}$ and $v=\left\{v_{i}\right\} ; 1=\{1\}$; and set 
then we have:

$$
\begin{gathered}
M=\hat{M}(0) \quad(=M(\infty)), \\
\beta=\lim _{\lambda \rightarrow 0}\langle\lambda \hat{\psi}(\lambda), 1\rangle \quad\left(=\psi_{*}(\infty)\right),
\end{gathered}
$$

$$
I+M U(\lambda) \text { and } I+U(\lambda) M \text { are both invertible for every } \lambda \text {; }
$$

$$
\langle M \beta, 1\rangle \leqslant 1
$$

The last inequality is equivalent to the Corollary to Theorem 6.6.

The full converse of the above will now be proved. We are given $Q$ on $\mathbf{I} \times \mathbf{I}$ to begin with satisfying Assumption A, from which $\Phi$ is defined. Let $\left\{l^{a}, a \in \mathbf{A}\right\}$ be a finite set of exit solutions and $\left\{\psi^{\tilde{a}}, \tilde{a} \in \tilde{\mathbf{A}}\right\}$ a finite set of entrance solution for $\Phi$; and define $U(\lambda)$ as in (8.3). Let $M$ be an $\mathbf{A} \times \tilde{\mathbf{A}}$ matrix with nonnegative constant elements satisfying (8.19) and (8.20). Now define $\hat{\bigcap}(\lambda)$ by (8.18).

THEOREM 8.3. $\hat{\Pi}(\lambda)$ is the Laplace transform of a substochastic transition matrix with $Q$ as its initial derivative matrix; and every such $\hat{\prod}(\lambda)$ can be constructed in this way under Assumptions A, B', $\tilde{\mathrm{A}}, \tilde{\mathrm{B}}^{\prime}$ and $\mathrm{C}$.

Proof. We have already proved the second part of the theorem.

The calculations for the first part will be briefly indicated, omitting the * on the Laplace transforms. We shall first verify the resolvent equation for $\Pi$ :

$$
\prod(\mu)-\Pi(\lambda)=(\lambda-\mu) \prod(\lambda) \prod(\mu) \quad\left(0<{ }_{\mu}^{\lambda}<\infty\right)
$$

We being by writing down similar equations for $\Phi, l$ and $\psi$ :

$$
\begin{gathered}
\Phi(\mu)-\Phi(\lambda)=(\lambda-\mu) \Phi(\lambda) \Phi(\mu), \\
l(\mu)-l(\lambda)=(\lambda-\mu) \Phi(\lambda) l(\mu), \\
\psi(\mu)-\psi(\lambda)=(\lambda-\mu) \psi(\lambda) \Phi(\mu),
\end{gathered}
$$

the last two being the double Laplace transforms of (4.4) and (7.18). Next, we define $\theta^{\tilde{a} a}(t)\left(^{1}\right)$ as in $(6.10)$ to be $\sum_{i} \psi_{i}^{\tilde{a}}(s) l_{i}^{a}(t-s), \hat{\theta}^{\tilde{a} a}(\lambda)$ to be its Laplace transform and $\Theta(\lambda)=\left(\tilde{\theta^{\alpha} a}(\lambda)\right),(\tilde{a}, a) \in \tilde{\mathbf{A}} \times \mathbf{A}$. Set also

$$
\begin{gathered}
\theta^{\tilde{a} a}(\lambda, \mu)=\sum_{i} \psi_{i}^{\tilde{a}}(\lambda) l_{i}^{a}(\mu)=\left\langle\psi^{\tilde{a}}(\lambda), l^{a}(\mu)\right\rangle, \\
\Theta(\lambda, \mu)=\left(\theta^{\tilde{a} a}(\lambda, \mu)\right) \quad((\tilde{a}, a) \in \tilde{\mathbf{A}} \times \mathbf{A}) .
\end{gathered}
$$

(1) This is the $\theta_{0}^{a a}(t)$ used momentarily in the second paragraph of the section. 
It follows from a computation based on Theorem 6.4 with $\eta$ replaced by $\psi$ that

$$
\Theta(\mu)-\Theta(\lambda)=(\lambda-\mu) \Theta(\lambda, \mu) \text {. }
$$

Finally, by the relation corresponding to the first equation in (6.11), we have

$$
U(\mu)-U(\lambda)=\Theta(\lambda)-\Theta(\mu) .
$$

Hence it follows from (8.17) that

$$
\begin{aligned}
& {[I+M U(\lambda)][M(\lambda)-M(\mu)][I+U(\mu) M]} \\
& =\boldsymbol{M}[\boldsymbol{I}+\boldsymbol{U}(\mu) \boldsymbol{M}]-[\boldsymbol{I}+\boldsymbol{M U}(\lambda)] \boldsymbol{M}=\boldsymbol{M}[U(\mu)-U(\lambda)] \boldsymbol{M} \\
& =M[\Theta(\lambda)-\Theta(\mu)] M=(\mu-\lambda) M \Theta(\lambda, \mu) M \\
& \text { or equivalently } \quad(\lambda-\mu) M(\lambda) \Theta(\lambda, \mu) M(\mu)=M(\mu)-M(\lambda) \text {. }
\end{aligned}
$$

Now we have, upon substitution from (8.18):

$$
\begin{gathered}
\prod(\lambda) \prod(\mu)=\Phi(\lambda) \Phi(\mu)+l(\lambda) M(\lambda) \psi(\lambda) \Phi(\mu)+\Phi(\lambda) l(\mu) M(\mu) \psi(\mu) \\
+l(\lambda) M(\lambda) \Theta(\lambda, \mu) M(\mu) \psi(\mu) .
\end{gathered}
$$

Hence using (8.22), (8.23), (8.24), and (8.26), we have

$$
\begin{aligned}
& (\lambda-\mu) \prod(\lambda) \prod(\mu) \\
& \quad=\Phi(\lambda)-\Phi(\mu)+l(\lambda) M(\lambda)[\psi(\mu)-\psi(\lambda)]+[l(\mu)-l(\lambda)] M(\mu) \psi(\mu) \\
& \quad+l(\lambda)[M(\mu)-M(\lambda)] \psi(\mu) \\
& =\Phi(\mu)+l(\mu) M(\mu) \psi(\mu)-\Phi(\lambda)-l(\lambda) M(\lambda) \psi(\lambda) \\
& =\prod(\mu)-\prod(\lambda) .
\end{aligned}
$$

Thus (8.21) is true. Next, we have by the relations corresponding to (6.16) with $\eta$ replaced by $\psi$ :

$$
\langle\lambda \psi(\lambda), 1\rangle=U(\lambda) 1+\beta .
$$

Hence it follows from (8.12), (8.17) and (8.20) that

$$
\begin{aligned}
\langle\lambda \xi(\lambda), \mathbf{l}\rangle & =M(\lambda)[U(\lambda) \mathbf{1}+\beta] \\
& =[I+M U(\lambda)]^{-1}[M U(\lambda)+M \beta] \\
& \leqslant[I+M U(\lambda)]^{-1}[M U(\lambda)+I] \mathbf{1}=\mathbf{1},
\end{aligned}
$$

and consequently for each $i \in I$, if $\Pi_{i}$ and $\Phi_{i}$ denote the $i$ th rows of $\Pi$ and $\Phi$ :

$$
\begin{aligned}
\left\langle\lambda \prod_{i}(\lambda), 1\right\rangle & =\left\langle\lambda \Phi_{i}(\lambda), 1\right\rangle+\sum_{a} l_{i}^{a}(\lambda)\left\langle\lambda \xi^{a}(\lambda), 1\right\rangle \\
& \leqslant 1-l_{i}(\lambda)+\sum_{a} l_{i}^{a}(\lambda)=1 .
\end{aligned}
$$


Thus $\Pi(\lambda)$ is substochastic, and is stochastic if, and only if, $M \beta=1$. Theorem 8.3 is proved.

The condition (8.19) can be made more explicit in particular cases. The following theorem is due to Feller [7; Theorem 14.1].

THEOREM 8.4. Suppose $U(\infty)<\infty$; then every $\widehat{\prod}(\lambda)$ can be constructed in the following way. Choose an $\mathbf{\Lambda} \times \tilde{\mathbf{\Lambda}}$ matrix $N$ with nonnegative constant elements satisfying the condition

set

$$
\begin{gathered}
N[U(\infty) 1+\beta] \leqslant 1 \\
M(\lambda)=\{I-N[U(\infty)-U(\lambda)]\}^{-1} N,
\end{gathered}
$$

and define $\hat{\Pi}(\lambda)$ by (8.18).

Proof. We prove only the necessity of (8.28) and (8.29); their sufficiency can be verified as in the preceding proof. Let us rewrite (8.7) as

$$
M(\lambda)=[I-M(\lambda) U(\lambda)] M .
$$

Letting $\lambda \rightarrow \infty$ and writing $N$ for $M(\infty)$, we have

$$
N=[I-N U(\infty)] M
$$

It follows from the condition (8.28) that $N U(\infty)$ is substochastic, and consequently each row of $N[U(\infty)-U(\lambda)]$ has a sum which is strictly less than one since the vanishing of a row sum in $N U(\lambda)$ implies that of the corresponding row sum in $N U(\infty)$. Hence the matrix

$$
I-N[U(\infty)-U(\lambda)]
$$

is invertible. The preceding argument is taken from Feller [7]. Now we have by (8.31),

$$
I-N[U(\infty)-U(\lambda)]=[I-N U(\infty)][I+M U(\lambda)]
$$

By Corollary 2 to Theorem 8.2, the second factor on the right side of (8.33), as well as the product, is invertible. Hence the first factor is also invertible by elementary matrix theory. We conclude by (8.17), (8.31) and (8.33) that

$$
\begin{aligned}
M(\lambda) & =[I+M U(\lambda)]^{-1} M=[I+M U(\lambda)]^{-1}[I-N U(\infty)]^{-1} N \\
& =\{I-N[U(\infty)-U(\lambda)]\}^{-1} N
\end{aligned}
$$

Next, we have from (8.31), $\quad M=[I-N U(\infty)]^{-1} N$

6-632932 Acta mathematica. 110. Imprimé le 16 octobre 1963. 
and consequently (8.20) becomes

which is (8.28).

$$
N \beta \leqslant[I-N U(\infty)] 1
$$

Note that if we write properly $\hat{M}(\lambda)$ for the $M(\lambda)$ above and use $M(t)$ as in Theorem 7.4, we have $M=\hat{M}(0)=M(\infty)$ and $N=\hat{M}(\infty)=M(0+)$, and we infer that the two sets $\left\{\zeta^{a}\right\}$ and $\left\{\eta^{a}\right\}, a \in A$, in (7.34) and (7.35) are linear combinations of each other. Apart from this additional information, the proof of Theorem 8.4 is unnecessarily complicated. Indeed (8.29) is a special case of our earlier Theorem 5.5, as to be shown now. By Theorem 7.5, the hypothesis that $U(\infty)<\infty$ implies that case (ii) in Theorem 5.5 occurs. By (7.34), we have $\hat{\zeta}(\lambda)=N \hat{\psi}(\lambda)$ where $N$ is as before. Using the notation in (5.27) and in the proof of Theorem 8.3, we have

$$
\Lambda(\lambda)=N \Theta(\lambda)=N[U(\infty)-U(\lambda)]
$$

the last equation being a consequence of (8.25). Substituting into (5.27), we obtain (8.29) by comparison with (8.12). Theorem 8.4 is proved.

The case where the matrix $U(\infty)$ contains infinite elements will now be sketched following Feller. A diagonal element of the matrix $I-M(\lambda) U(\lambda)$ is of the form

$$
1-\lambda \sum_{i} \hat{\xi}_{i}^{a}(\lambda) L_{i}^{a}(\infty)=\lambda \int_{0}^{\infty} e^{-\lambda t}\left[1-\sum_{i} \xi_{i}^{a}(t) L_{i}^{a}(\infty)\right] d t
$$

hence positive unless $L_{i}^{a}(\infty)=1$ for each $i \in \mathbf{I}^{a}$. It is easy to see that this is impossible under Assumption C. Hence we can write

$$
I-M(\lambda) U(\lambda)=D(\lambda)[I-\bar{S}(\lambda)]
$$

where $D(\lambda)$ is the diagonal part of the matrix on the left side of (8.35) and $\bar{S}(\lambda)$ has zero elements on the diagonal. Now define $\bar{M}(\lambda)$ by

$$
\bar{M}(\lambda)=[D(\lambda)]^{-1} M(\lambda)=[I-\bar{S}(\lambda)] M,
$$

where the second equation follows from (8.30). Letting $\lambda_{n} \rightarrow \infty$ so that

$$
\lim _{n \rightarrow \infty} \bar{M}\left(\lambda_{n}\right)=\bar{M}, \quad \lim _{n \rightarrow \infty} \bar{S}\left(\lambda_{n}\right)=\bar{S},
$$

we obtain

$$
\bar{M}=[I-\bar{S}] M
$$

Thus $\bar{M}$ and $\bar{S}$ take the place of $N$ and $N U(\infty)$ respectively in (8.31). Substituting (8.6) into (8.38), we obtain

$$
\bar{M}=[I-\bar{S}][I+M U(\lambda)] M(\lambda)=[I-\bar{S}+\bar{M} U(\lambda)] M(\lambda) .
$$


Let us classify the indices in $\mathbf{A}$ according to the substochastic matrix $\bar{S}$, in a similar way as in $\S 5$. Just as there, the matrix $I-\bar{S}$ is invertible unless there exists an essential class $C$ such that for every $a$ in $C$, the ath row in $\bar{S}$ has sum equal to one. Since $\bar{S}$ is zero on the diagonal, such a class $C$ must contain more than one index. It follows from (8.38) that if $M_{C}, \bar{M}_{C}$ denote the restrictions of $M, \bar{M}$ to $C \times \tilde{\mathbf{A}}$, but $\bar{S}_{C},(I-\bar{S})_{C}$ those of $\bar{S}, I-\bar{S}$ to $C \times C$, we have

$$
M_{C}=\bar{M}_{C}+\bar{S}_{C} M_{C} \geqslant \bar{S}_{C} M_{C}
$$

Now a general theorem about discrete paramater Markov chains states that an excessive (superregular) function bounded below on a recurrent class is a constant. (In the case of a finite class as here, a simple algebraic proof is obtained by considering the minimum value of the function.) Applying this to (8.40) we infer that equality holds in (8.40) so that $\bar{M}_{C}=0$, and consequently we have by (8.39):

$$
[I-\bar{s}]_{C} M_{C}(\lambda)=0
$$

It follows from (8.12) that, if $\hat{\xi}_{C}(\lambda)$ denotes the restriction of $\hat{\xi}(\lambda)$ to $C$ :

$$
[I-\bar{S}]_{C} \hat{\xi}_{C}(\lambda)=[I-\bar{S}]_{C} M_{C}(\lambda) \hat{\psi}(\lambda)=0
$$

and so

$$
\hat{\xi}_{C}(\lambda)=\bar{S}_{C} \hat{\xi}_{C}(\lambda)
$$

Applying again the theorem just cited, we see that $\hat{\xi}(\lambda)$ is constant on $C$. This being true for every $\lambda$, we conclude that $\xi^{a}(t) \equiv \xi^{b}(t)$ for every $a$ and $b$ in $C$. Thus the boundary points $\infty^{a}$ for $a$ in $C$ are all indistinguishable from each other. If this eventuality is excluded, then $I-\bar{S}$ is invertible, and so is $I-\bar{S}+\bar{M} U(\lambda)$. We have therefore proved the following result.

THEOREM 8.5. If all boundary points are distinguishable from each other, then we have

$$
M(\lambda)=[I-\bar{S}+\bar{M} U(\lambda)]^{-1} \bar{M}
$$

This was proved by Feller under the superfluous assumption that every element of $U(\lambda)$ be positive. $\left(^{1}\right)$ For the consequent construction theorem similar to Theorem 8.4 above, we refer to Feller [7].

(1) I am indebted to David Williams for a verification of Feller's theorem by a purely algebraic method, which leads to the disposition above. 


\section{§ 9. The One Exit Case}

We make Assumptions $\mathrm{A}, \mathrm{B}$ and $\mathrm{C}$ and the further assumption that the set $\mathrm{A}$ in Assumption B consists of one element only. The index $a$ corresponding to this element will be omitted, thus e.g., $l_{i}(t)=l_{i}^{a}(t)$.

In this case we have

$$
\begin{aligned}
& \sigma(t)=\sum_{i} g_{i} l_{i}(t)=\sum_{i} \eta_{i}(t) L_{i}(\infty), \\
& u(\lambda)=\lambda \hat{\sigma}(\lambda)=\lambda \int_{0}^{\infty} e^{-\lambda t} \sigma(t) d t
\end{aligned}
$$

and (8.1) reduces to

$$
\hat{\eta}(\lambda)=[1+u(\lambda)] \hat{\xi}(\lambda)
$$

or

$$
\hat{\xi}(\lambda)=m(\lambda) \hat{\eta}(\lambda)
$$

where

$$
m(\lambda)=\frac{1}{1+u(\lambda)}
$$

It follows from Theorem 8.1 with $M=1$ that $(1+u(\lambda))^{-1}$ is a completely monotonic function of $\lambda$. We have by (6.16),

$$
1+u(\lambda)=1-\eta_{*}(\infty)+\lambda \hat{\eta}_{*}(\lambda)
$$

and

$$
\lambda \sum_{j \in \mathbf{I}} \hat{\xi}_{j}(\lambda)=\frac{\lambda \hat{\eta}_{*}(\lambda)}{1+u(\lambda)}=\frac{\lambda \hat{\eta}_{*}(\lambda)}{\lambda \hat{\eta}_{*}(\lambda)+1-\eta_{*}(\infty)}
$$

It follows that $\sum_{j \in \mathbf{I}} \xi_{j}(t)=\mathbf{l}$ or that $\left(p_{i j}\right),(i, j) \in \mathbf{I} \times \mathbf{I}$, is stochastic if and only if $\eta_{*}(\infty)=1$. In general

$$
\lambda \hat{\xi}_{\theta}(\lambda)=\frac{1-\eta_{*}(\infty)}{1+u(\lambda)}
$$

Hence we have

$$
\begin{gathered}
\xi_{\theta}(0)=\frac{1-\eta_{*}(\infty)}{1-\eta_{*}(\infty)+\eta_{*}(0)}, \\
\xi_{\theta}(\infty)=1-\eta_{*}(\infty) .
\end{gathered}
$$

It is possible to extend the equation (6.7) to $\xi_{\theta}$ as follows. Since

$$
\xi_{\theta}(s+t)-\xi_{\theta}(s)=\sum_{i \in \mathbf{I}} \xi_{i}(s) p_{i \theta}(t)=\sum_{i \in \mathbf{I}} \xi_{i}(s) \int_{0}^{t} l_{i}(u) \xi_{\theta}(t-u) d u
$$

we have

$$
\int_{0}^{\infty}\left[\xi_{\theta}(s+t)-\xi_{\theta}(s)\right] d s=\int_{0}^{t} \sigma(u) \xi_{\theta}(t-u) d u
$$


Since $\xi_{\theta}$ is absolutely continuous, the left member above is equal to

$$
\int_{0}^{\infty} \int_{s}^{s+t} \xi_{\theta}^{\prime}(u) d u d s=\int_{0}^{t} u \xi_{\theta}^{\prime}(u) d u+t \int_{t}^{\infty} \xi_{\theta}^{\prime}(u) d u=t \xi_{\theta}(\infty)-\int_{0}^{t} \xi_{\theta}(u) d u .
$$

It follows that $\quad t \xi_{\theta}(\infty)=\int_{0}^{t} \xi_{\theta}(s) d s+\int_{0}^{t} \sigma(s) \xi_{\theta}(t-s) d s$

Thus to extend (6.7) to $\xi_{\theta}$ we should set $\eta_{\theta}(t) \equiv \xi_{\theta}(\infty)$.

The functions $\eta_{j}(\cdot)$ in Theorem 6.2 can be decomposed into two parts. Letting $s \downarrow 0$ in $(6.5)$ we have

$$
\sum_{i} \eta_{i}(0) f_{i j}(t) \leqslant \eta_{j}(t)
$$

If we set

$$
\bar{\eta}_{j}(t)=\eta_{j}(t)-\sum_{i} \eta_{i}(0) f_{i j}(t)
$$

then $\left\{\bar{\eta}_{i}(\cdot)\right\}$ is an entrance solution for $\Phi$ satisfying $\bar{\eta}_{i}(0)=0$. Consequently, we have by Theorem 6.7,

$$
\sum_{i} \bar{\eta}_{i}(t) q_{i j}=0
$$

These results check with Reuter [13]. It is to be noted that Reuter's analytical assumption implies our Assumption C, unless I consists of one П-recurrent class.

The function $m(\lambda)$ in $(9.1)$ is of interest. Note that

$$
u(\lambda)=\int_{0}^{\infty} \lambda e^{-\lambda t} d t \int_{t}^{\infty} \theta(s) d s=\int_{0}^{\infty}\left(1-e^{-\lambda s}\right) \theta(s) d s
$$

and by (6.7) and Corollary 2 to Theorem 6.3:

$$
\begin{aligned}
\int_{0}^{1} s \theta(s) d s & =\int_{0}^{1} d s \int_{0}^{s} \sum_{i} \eta_{i}(u) l_{i}(s-u) d u=\int_{0}^{1} \sum_{i} \eta_{i}(u) L_{i}(1-u) d u \\
& \leqslant \int_{0}^{1} \eta_{*}(u) d u \leqslant t+\int_{0}^{t} \sigma(s) d s<\infty
\end{aligned}
$$

Hence the last member of $(9.2)$ is the negative Laplace transform of an infinitely divisible distribution on $\mathbf{T}$. Precisely, there is a process $\{\boldsymbol{Y}(v), v \in \mathbf{T}\}$ with stationary independent positive increments such that

$$
\mathbf{E}\left(e^{-\lambda Y(v)}\right)=\exp \left[v \int_{0}^{\infty}\left(e^{-\lambda s}-1\right) \theta(s) d s\right]
$$


It follows that

$$
m(\lambda)=\int_{0}^{\infty} e^{-v[1+u(\lambda)]} d v=\mathbf{E}\left(e^{-\lambda Y(\mu)}\right)
$$

where $\mu$ is a random variable with the distribution function $e_{1}$ and independent of the process $\{\boldsymbol{Y}(v)\}$.

In the particular case where $\int_{0}^{\infty} \theta(s) d s=\sigma(0)<\infty$, we set

$$
F(t)=\frac{1}{\sigma(0)} \int_{0}^{t} \theta(s) d s
$$

The following theorem is easily proved.

THкOR $\mathbf{9}$ 9.1. Let the random variable $\mu$ be as described above and let the random variable $y$ have the geometric distribution given as follows:

$$
\mathbf{P}\{v=n\}=\left(\frac{\sigma(0)}{1+\sigma(0)}\right)^{n} \frac{1}{1+\sigma(0)}, \quad n \in \mathbf{N} .
$$

Let $\left\{y_{n}, n \in \mathbf{N}\right\}$ be a sequence of independent random variables having the common distribution function $F$ in (9.5) and independent of $\nu$. Then $Y(\mu)$ and $\sum_{n=1}^{v} y_{n}$ have the same distribution.

The matrix generalization of this theorem is implicit in Theorem 8.4; see also the discussion at the end of $\S 5$. For the case where the matrix $\left(\sigma^{\tilde{a} a}(0)\right)$ is infinite on the diagonal and finite elsewhere see Neveu [10], [11]. The extent to which his results generalize Theorem 9.1 is not clear. The representation (9.4) must be intimately related to Paul Lévy's "local time" (see [9]), but again the exact connection is not clear.

\section{Appendix}

The following theorem, under the additional assumption of (2.4) with equality, was first proved with probabilistic methods by D. G. Austin; a simplified version by the present author is given as Theorem II.5.2 in [1]. A simpler analytic proof was later obtained by D. Ornstein; it is given as Theorem II.1.5 in [1]. The present proof, without the assumption (2.4), is a modification of the latter.

THEOREM 10.1. Let $\left(p_{i j}\right),(i, j) \in \mathbf{I} \times \mathbf{I}$, be a matrix of functions on $\mathbf{T}$ satisfying (2.1), (2.2) and (2.3). Then each $p_{i j}(\cdot)$ is either identically zero or never zero.

Proof. Suppose $t_{0}>0$ and $p_{i j}\left(t_{0}\right)=0$. Let $N$ be a positive integer and $t_{0}=2 N s$. Define 


$$
\begin{aligned}
& C_{m}=\left\{k: p_{i k}(m s)=0\right\}, \\
& C_{m}-C_{m+1}=D_{m+1} \quad(m \geqslant 0) .
\end{aligned}
$$

Then $C_{0}=\mathbf{I}-\{i\}, C_{m} \searrow$, and $j \in C_{2 N}$. Let us put

$$
\begin{aligned}
& u(m, n)=\sum_{k \in C_{m}} p_{i k}(n s) p_{k j}(4 N s-n s) \\
& v(m, n)=\sum_{k \in D_{m}} p_{i k}(n s) p_{k j}(4 N s-n s)
\end{aligned}
$$

We have $\quad u(m, 0)=0 \quad(0 \leqslant m) ; \quad u(m, 4 N)=p_{i j}(4 N s) \quad(0 \leqslant m \leqslant 2 N)$.

By [3; Theorem 1], each $p_{i j}$ is continuous in $\mathbf{T}$; hence by Dini's theorem, the series

$$
\sum_{k \in \mathbf{I}} p_{i k}(t) p_{k j}\left(2 t_{0}-t\right)=p_{i j}\left(2 t_{0}\right)
$$

converges uniformly in $t \in\left[0,2 t_{0}\right]$. Since the $D_{m}$ 's are disjoint (possibly void) and

$$
\sum_{m=0}^{\infty} v(m, n) \leqslant \sum_{k \in \mathbf{I}} p_{i k}(n s) p_{k j}(4 N s-n s)
$$

it follows from the uniform convergence of the series in (10.1), that

$$
\sum_{m=0}^{\infty} v(m, n) \text { converges uniformly in } n, 0 \leqslant n \leqslant 4 N \text {. }
$$

We have by the definitions:

$$
u(m, n+1)-v(m+1, n+1)=\sum_{k \in C_{m}+1}\left(\sum_{l \in \mathbf{I}} p_{i l}(n s) p_{l k}(s)\right) p_{k j}(4 N s-n s-s) .
$$

If $k \in C_{m+1}$ and $p_{l k}(s)>0$, then $l \in C_{m}$; for otherwise $p_{i k}(m s+s) \geqslant p_{i l}(m s) p_{l k}(s)>0$ and $k$ would not belong to $C_{m+1}$. Hence in the double sum in (10.3) we need only sum $l$ over $C_{m}$, and consequently

$$
\begin{aligned}
u(m, n+1)-v(m+1, n+1) & \leqslant \sum_{l \in C_{m}} p_{i l}(n s) \sum_{k \in \mathbf{I}} p_{l k}(s) p_{k j}(4 N s-n s-s) \\
& =\sum_{l \in C_{m}} p_{i l}(n s) p_{l j}(4 N s-n s)=u(m, n) .
\end{aligned}
$$

Summing over $n$ we obtain

$$
p_{i j}(4 N s)=u(m, 4 N) \leqslant \sum_{n=0}^{4 N-1} v(m+1, n+1) .
$$

This being true for $0 \leqslant m \leqslant 2 N$, we infer that 


$$
p_{i j}\left(2 t_{0}\right) \leqslant \frac{1}{N} \sum_{n=0}^{4 N-1} \sum_{m=N}^{2 N-1} v(m+1, n+1) \leqslant 4 \max _{1 \leqslant n \leqslant 4 N} \sum_{m=N+1}^{2 N} v(m, n)
$$

As $N \rightarrow \infty$, the last member above converges to zero by $(10.2)$, and so $p_{i j}\left(2 t_{0}\right)=0$. Repeating this argument, we see that $p_{i j}\left(2^{n} t_{0}\right)=0$ for every positive integer $n$ and consequently $p_{i j}(t) \equiv 0$, since $p_{i j}(t)>0$ implies $p_{i j}\left(t^{\prime}\right)>0$ for $t^{\prime}>t$ trivially. The theorem is proved.

CoRolla RY. Let $\left\{\xi_{j}(\cdot), j \in \mathbf{I}\right\}$ be nonnegative functions on $\mathbf{T}^{\mathbf{0}}$ satisfying either

or

$$
\begin{array}{ll}
\xi_{j}(t)=\sum_{i \in \mathbf{I}} \xi_{i}(s) p_{i j}(t-s) & (0<s<t), \\
\xi_{i}(t)=\sum_{j \in \mathbf{I}} p_{i j}(t-s) \xi_{j}(s) & (0<s<t),
\end{array}
$$

for every $t \in \mathbf{T}^{\mathbf{0}}$. Then each $\xi_{j}(\cdot)$ is either identically zero or never zero in $\mathbf{T}^{\mathbf{0}}$.

Proof. Theorem 10.1 being symmetric in the pair of indices $(i, j)$, we need only prove the first form ' of the Corollary. If for some $t>0$ we have $\xi_{j}(t)>0$, then for any $\delta: 0<\delta<t$, there exist $s: 0<s<\delta$, and $i \in I$ such that $\xi_{i}(s)>0$ and $p_{i j}(t-s)>0$. Hence by the theorem, $p_{i j}(\delta-s)>0$ and so $\xi_{j}(\delta) \geqslant \xi_{i}(s) p_{i j}(\delta-s)>0$. Since $\delta$ is arbitrary $\xi_{j}(\cdot)>0$ in $\mathbf{T}^{0}$, proving the corollary.

It follows from the Corollary that each function such as $k, l, \xi, \eta, \zeta, \psi$ in the text, which is a member of an exit or entrance solution for a standard transition matrix ( $\Pi$ or $\Phi$ ), has the always-or-never-zero property. The result can be generalized at once to a measurable transition matrix (see the last paragraph of p. 122 in [1]).

\section{References}

[1]. Chung, K. L., Markov Chains with Stationary Transition Probabilities. Springer, Berlin Göttingen, Heidelberg, 1960.

[2]. —- On last exit times. Illinois J. Math., 4 (1960), 629-39.

[3]. - - Probabilistic Methods in Markov Chains. Fourth Berkeley Symposium on Mathematical Statistics and Probability, Vol. II, 35-56, 1961. University of California Press, Berkeley and Los Angeles.

[4]. - - On the Martin boundary for Markov chains. Proc. Nat. Acad. Sci., 48 (1962), 963-968.

[5]. Dоов, J. L., Discrete potential theory and boundaries. J. Math. Mech., 8 (1959), 433-458.

[6]. Feller, W., Boundaries induced by positive matrices. Trans. Amer. Math. Soc., 83 (1956), 19-54.

[7]. - On boundaries and lateral conditions for the Kolmogorov differential equations. Ann. of Math., 65 (1957), 527-570; “Notes", ibid., 68 (1958), 735-736. 
[8]. Hunt, G. A., Markoff chains and Martin boundaries. Illinois J. Math., 4 (1960), 313-340.

[9]. LÉvy, P., Systèmes markoviens et stationnaires; cas dénombrable. Ann. École Norm. (3), 68 (1951), 327-381.

[10]. NeveU, J., Une généralisation des processus à accroisements croissants indépendants. Abh. Math. Mem. Univ. Hamburg, 25 (1961), 36-61.

[11]. - - Lattice methods and submarkovian processes. Fourth Berkeley Symposium on Mathematical Statistics and Probability, Vol. II, 347-391, 1961. University of California Press, Berkeley and Los Angeles.

[12]. Reuter, G. E. H., Denumerable Markov processes and the associated contraction semiproups on $l$. Acta Math., 97 (1957), 1-46.

[13]. - - Denumerable Markov processes (II). J. London Math. Soc., 34 (1959), 81-91.

[14]. — Denumerable Markov processes (III). Ibid., 37 (1962), 63-73.

Received March 8, 1963 